\title{
Coordinated reset stimulation in a large-scale model of the STN-GPe circuit
}

\author{
Martin Ebert ${ }^{1,2}$, Christian Hauptmann ${ }^{1 *}$ and Peter A. Tass ${ }^{1,3,4}$ \\ ${ }^{1}$ Institute of Neuroscience and Medicine - Neuromodulation, Juelich Research Center GmbH, Juelich, Germany \\ 2 Department of Physics, Institute of Nuclear Physics, University of Cologne, Cologne, Germany \\ ${ }^{3}$ Department of Neurosurgery, Stanford University, Stanford, CA, USA \\ ${ }^{4}$ Department of Neuromodulation, University of Cologne, Cologne, Germany
}

\section{Edited by:}

David Hansel, University of Paris,

France

Reviewed by:

Kenji Morita, The University of Tokyo, Japan

Jonathan Rubin, University of Pittsburgh, USA

Alessandro Torcini, Consiglio

Nazionale delle Ricerche, Italy

*Correspondence:

Christian Hauptmann, Institute of Neuroscience and Medicine Neuromodulation, Juelich Research Center GmbH, Leo-Brandt Strasse 52425 Juelich, Germany

e-mail: c.hauptmann@fz-juelich.de
Synchronization of populations of neurons is a hallmark of several brain diseases. Coordinated reset (CR) stimulation is a model-based stimulation technique which specifically counteracts abnormal synchrony by desynchronization. Electrical CR stimulation, e.g., for the treatment of Parkinson's disease (PD), is administered via depth electrodes. In order to get a deeper understanding of this technique, we extended the top-down approach of previous studies and constructed a large-scale computational model of the respective brain areas. Furthermore, we took into account the spatial anatomical properties of the simulated brain structures and incorporated a detailed numerical representation of $2 \cdot 10^{4}$ simulated neurons. We simulated the subthalamic nucleus (STN) and the globus pallidus externus (GPe). Connections within the STN were governed by spike-timing dependent plasticity (STDP). In this way, we modeled the physiological and pathological activity of the considered brain structures. In particular, we investigated how plasticity could be exploited and how the model could be shifted from strongly synchronized (pathological) activity to strongly desynchronized (healthy) activity of the neuronal populations via CR stimulation of the STN neurons. Furthermore, we investigated the impact of specific stimulation parameters especially the electrode position on the stimulation outcome. Our model provides a step forward toward a biophysically realistic model of the brain areas relevant to the emergence of pathological neuronal activity in PD. Furthermore, our model constitutes a test bench for the optimization of both stimulation parameters and novel electrode geometries for efficient CR stimulation.

Keywords: deep brain stimulation, high-performance computing, coordinated reset stimulation, electrode design

\section{INTRODUCTION}

Several brain disorders are associated with abnormal neuronal synchronization. For example, epileptic seizures are characterized by an abnormally synchronized firing of neurons during the epileptic process (Lehnertz et al., 2009). Parkinson's disease (PD) is another pathological condition of the central nervous system, which is associated with abnormal neuronal synchronization in specific brain regions (Lenz et al., 1994; Nini et al., 1995; Plenz and Kital, 1999). The occurrence of resting tremor with frequencies between 4 and $9 \mathrm{~Hz}$ in $\mathrm{PD}$ is correlated to pathological synchronization of particular neuronal populations located in the thalamus and basal ganglia (Deuschl et al., 2000; Bevan et al., 2002; Mallet et al., 2008; Smirnov et al., 2008; Tass et al., 2010), which actually seem to drive the tremor (Smirnov et al., 2008; Tass et al., 2010). In contrast, in the healthy state these populations fire in an uncorrelated manner (Nini et al., 1995; Brown et al., 2001; Fahn, 2008).

An established therapy for patients with medically refractory PD is electrical deep brain stimulation (DBS) (Benabid et al., 1991; Rodriguez-Oroz et al., 2005; Deuschl et al., 2006). Today the field of application of DBS is expanding. For instance, DBS has recently been evaluated in clinical trials in patients suffering from treatment-resistant depression, and first-in-man studies have been conducted for the treatment of Tourette's syndrome (Anderson et al., 2012; Cannon et al., 2012). Despite positive clinical results, the understanding of the mechanisms of action underlying the therapeutic effects of DBS is still a matter of debate (Grill and McIntyre, 2001; Volkmann, 2004; Anderson et al., 2012).

For DBS depth electrodes are implanted in particular target areas during high precision stereotactic surgery (Voges et al., 2002; Coffey, 2009). For the treatment of PD, the STN is the most commonly used target for DBS (Rodriguez-Oroz et al., 2005; Deuschl et al., 2006). This small lens-shaped brain structure is part of the basal ganglia, which are important for voluntary movement control (Nini et al., 1995). The electrodes mostly used for implantation comprise four cylindrical contacts, which are made of a Pt-Ir alloy. Each contact can individually be activated for stimulation (Coffey, 2009). Extension cables connect the electrodes to the pacemaker, which is implanted below the patient's collarbone and serves as counter-pole during stimulation. The standard DBS algorithm is high-frequency 
(HF) permanent stimulation, where a biphasic current pulse train is continuously administered via the implanted electrode contact(s). Stimulation frequencies of $100-180 \mathrm{~Hz}$ and amplitudes varying between 0 and $10 \mathrm{~V}$ have clinically proven to be effective (Moro et al., 2002; Volkmann et al., 2002). The mechanisms of DBS are still matter of debate (McIntyre et al., 2004c). One hypothesis is, that HF DBS leads to a suppression of activity in the target structure and thus mimics lesioning effects in a reversible way (McIntyre et al., 2004c; Volkmann, 2004; Kühn et al., 2008; Montgomery and Gale, 2008; Pyragas et al., 2013). However, there is evidence for other non-local mechanisms (McIntyre et al., 2004c; Gradinaru et al., 2009). Rubin et al. (2012) gives a good review of the complex mechanisms of DBS based on experimental and numerical observations.

HF DBS treatment allows for a significant reduction of medication (Deuschl et al., 2006). However, there is a relevant nonresponder rate, for instance, tremor suppression is non-effective in $15 \%$ of the patients, and in $73 \%$ of the patients HF DBS is noneffective with respect to axial symptoms (Limousin et al., 1999; Volkmann, 2004). In fact, not all symptoms are equally reduced (Rodriguez-Oroz et al., 2005), some patients show side effects such as worsening speech, reduced verbal fluency, and weight gain affect an important number of patients (Voges et al., 2002). Some of these side effects may result from current spread to remote structures or improper electrode placement, but the underlying reasons for the occurrence of side effects are still not sufficiently understood (Voges et al., 2002).

To overcome limitations of HF DBS, with methods from nonlinear dynamics and statistical physics, stimulation techniques have been developed, which specifically counteract pathological neuronal synchronization by desynchronization (Tass, 1999). Coordinated reset (CR) stimulation is a robust desynchronization technique which can be applied in an open loop as well as closed loop mode (Tass, 2003b,c).

$\mathrm{CR}$ aims at a therapeutic reshaping of neuronal connectivity by harnessing synaptic plasticity (e.g., spike timing-dependent plasticity, STDP) (Gerstner et al., 1996; Markram et al., 1997). Based on Shen et al. (2003) we hypothesize that STDP also occurs within the STN population. The goal of CR stimulation is to decrease the rate of coincidences, which - due to STDP - causes a decrease of the synaptic weights and, in turn, an unlearning of pathological connectivity and synchrony (Tass and Majtanik, 2006). Put otherwise, CR DBS aims at long-lasting effects that persist after cessation of stimulation (Tass and Majtanik, 2006; Tass and Hauptmann, 2007). First experimental in vitro studies in rat hippocampal slice and in vivo experiments in Parkinsonian monkeys confirmed that CR causes a long-lasting desynchronization (Tass et al., 2009) and reduction of symptoms (Tass et al., 2012b). By the same token, long-lasting and cumulative aftereffects of CR stimulation were observed in a proof of concept study in PD patients (Adamchic et al., 2014a). Computational studies showed that CR stimulation can be delivered both invasively and non-invasively (Popovych and Tass, 2012; Tass and Popovych, 2012). Accordingly, CR has successfully been applied to the treatment of tinnitus via acoustic stimulation (Tass and Popovych, 2012; Tass et al., 2012a; Silchenko et al., 2013; Adamchic et al., 2014b).
The biophysical mechanism of action of DBS is not yet sufficiently understood (Grill and McIntyre, 2001; Volkmann, 2004). In order to investigate how electrical stimulation affects neuronal tissue, computational models of the respective systems are useful tools (Rubin and Terman, 2004; Miocinovic et al., 2006; So et al., 2012). In the present study we focus on the effects of different electrical stimulation algorithms on the collective activity of a model neuronal network. So far, the mechanisms of CR stimulation have been studied utilizing neuron models with reduced complexity and a limited number of modeled neurons (Hauptmann et al., 2005; Tass and Hauptmann, 2009; Guo and Rubin, 2011; Lysyansky et al., 2011). Therefore, in this study we consider a sufficient number of neurons required to appropriately sample the stimulated volume in space and establish a computational platform for time consuming simulations taking into account the slow STDP-mediated dynamics.

The high number and density of neurons gave us the opportunity to investigate local and propagation effects of synchronization within the network. Hence, we constructed a large-scale model of the two structures hypothesized to be responsible for the pathological activity associated with PD. Our model contains a network of in total $2 \cdot 10^{4}$ neurons. In contrast to previous models, where the nodes of the simulated networks were placed on one- or two-dimensional (regular) lattices (Hauptmann et al., 2005; Guo and Rubin, 2011; Lysyansky et al., 2011), and similar to a previous study (Hauptmann and Tass, 2010) we arranged the neurons within a spatial network in order to replicate the three-dimensional structure of the simulated parts of the brain. We used a conductance based, and biophysically realistic model associated with physical dimensions for the individual neurons (Terman et al., 2002; Rubin and Terman, 2004).

With increased complexity, however, it becomes challenging to fully understand the dynamics of the system. Hence, we mainly based our model on experimentally constrained parameters and continued the top-down approach of previous studies (Hauptmann et al., 2005; Hauptmann and Tass, 2007; Maistrenko et al., 2007; Tass and Hauptmann, 2007, 2009; Hauptmann and Tass, 2009, 2010; Lysyansky et al., 2011; Popovych and Tass, 2012) by increasing the complexity of the considered model gradually. The model presented in this study can be considered as a step forward toward a biophysically realistic model of a major target area for DBS.

\section{METHODS}

We used Neural Simulation Tool (NEST) (version 2.0.0-rc4) (Gewaltig and Diesmann, 2007) to implement and perform the simulations presented in this study. The simulation code of the model network was implemented using the Python interface for NEST (Eppler et al., 2008). NEST allows for an implementation of three dimensional models of neuronal networks via the topology module (Plesser and Austvoll, 2009). We performed all simulations on the high-performance computer JUROPA at the Research Center Jülich, Germany. This supercomputer is composed of 3288 compute nodes, while each node comprises two Intel Xeon X55704 quad-core processors, resulting in 26304 processors in total available for computation. The supercomputer is equipped with a main memory capacity of $79 \mathrm{~TB}$ and 
provides 274.8 FLOPS performance, measured by the LINPACKbenchmark. Accordingly, each compute node has access to $24 \mathrm{~GB}$ of memory. This high amount of memory available per node allows for simulations with complex individual neurons and an online analysis of simulation data. The various computation nodes are linked via an InfiniBand quad data rate fat tree topology. The used Message Passing Interface (MPI) implementation was Parastation-MPI 5.0.25. The differential equations describing the dynamics of our model network were solved numerically utilizing an embedded Runge-Kutta-Fehlberg method of fourth order with adaptive step size control, which is implemented as part of the GNU Scientific Library (GSL) (version 1.14) (Fehlberg, 1970; Galassi et al., 2009).

\subsection{MODEL EQUATIONS}

In order to model the neuronal activity of the individual STN and GPe neurons, we used the model developed by Terman and Rubin (Terman et al., 2002; Rubin and Terman, 2004), which is a singlecompartment conductance based model.

$$
c_{\mathrm{m}} \frac{d v}{d t}=-I_{\mathrm{L}}-I_{\mathrm{K}}-I_{\mathrm{Na}}-I_{\mathrm{T}}-I_{\mathrm{Ca}}-I_{\mathrm{ahp}}-I_{\text {syn }}+I_{\text {stim }}+I_{\text {noise }}(1)
$$

The $\mathrm{Na}^{+}$and $\mathrm{K}^{+}$ionic currents $I_{\mathrm{Na}}, I_{\mathrm{K}}$ are responsible for the spike production. Besides those spike-producing currents a lowthreshold T-type $\mathrm{Ca}^{2+}$ current $I_{\mathrm{T}}$, a high-threshold $\mathrm{Ca}^{2+}$ current $I_{\mathrm{Ca}}$ along with a $\mathrm{K}^{+}$current $I_{\mathrm{ahp}}$, which kicks in after hyperpolarization, enter the equation. The intrinsic neuron dynamics is additionally influenced by synaptic inputs from connected STN and GPe neurons $I_{\text {syn }}$, the input from surrounding brain structures $I_{\text {noise, }}$ and an external stimulation current $I_{\text {stim. }}$ Via this stimulation current the influence of DBS on each single neuron is modeled. The various ionic currents are all expressed in $\mathrm{pA} / \mu \mathrm{m}^{2}$ and described by several equations:

$$
\begin{aligned}
I_{\mathrm{L}} & =g_{\mathrm{L}}\left[v-v_{\mathrm{L}}\right] \\
I_{\mathrm{K}} & =g_{\mathrm{K}} n^{4}\left[v-v_{\mathrm{K}}\right] \\
I_{\mathrm{Na}} & =g_{\mathrm{Na}} m_{\infty}^{3}(v) h\left[v-v_{\mathrm{Na}}\right] \\
I_{\mathrm{T}} & =g_{\mathrm{T}} a_{\infty}^{3}(v) b_{\infty}^{2}(r)\left[v-v_{\mathrm{Ca}}\right] \\
I_{\mathrm{Ca}} & =g_{\mathrm{Ca}} s_{\infty}^{2}(v) h\left[v-v_{\mathrm{Ca}}\right] \\
I_{\mathrm{ahp}} & =g_{\mathrm{ahp}}\left[v-v_{\mathrm{K}}\right] \frac{[\mathrm{Ca}]}{[\mathrm{Ca}]+k_{1}} \\
\frac{d[\mathrm{Ca}]}{d t} & =\Gamma\left(-I_{\mathrm{Ca}}-I_{\mathrm{T}}-k_{\mathrm{Ca}}[\mathrm{Ca}]\right)
\end{aligned}
$$

The equations for both STN and GPe neurons are very similar. Besides different parameter values, the only difference is the slightly simpler form of the low threshold $\mathrm{Ca}^{2+}$ current $I_{\mathrm{T}}=g_{\mathrm{T}} a_{\infty}^{3}(v) r\left[v-v_{\mathrm{Ca}}\right]$ for the GPe neurons. In our model the

\begin{tabular}{|c|c|c|c|}
\hline & $\mathrm{nS} / \mu \mathbf{m}^{2}$ & & $\mathrm{mV}$ \\
\hline$g\llcorner$ & 2.25 & $v_{\mathrm{L}}$ & -60.0 \\
\hline$g_{\mathrm{K}}$ & 45.0 & $v_{\mathrm{K}}$ & -80.0 \\
\hline$g_{\mathrm{Na}}$ & 37.5 & $v_{\mathrm{Na}}$ & 55.0 \\
\hline$g_{\mathrm{Ca}}$ & 0.5 & $v_{\mathrm{Ca}}$ & 140.0 \\
\hline$g_{\mathrm{ahp}}$ & 9.0 & $v_{\mathrm{sS}}$ & 0.0 \\
\hline \multirow[t]{2}{*}{$g_{\top}$} & 0.5 & $v_{\mathrm{sg}}$ & 0.0 \\
\hline & ms & & ms \\
\hline$\eta_{h}$ & 500.0 & $\xi_{h}$ & 1.0 \\
\hline$\eta_{n}$ & 100.0 & $\xi_{n}$ & 1.0 \\
\hline$\eta_{r}$ & 17.5 & $\xi_{r}$ & 40.0 \\
\hline$\vartheta_{h}$ & 0.75 & $\vartheta_{n}$ & 0.75 \\
\hline$\vartheta_{r}$ & 0.2 & & \\
\hline$\tau_{\mathrm{SS}}$ & 1.0 & $\tau_{\mathrm{gs}}$ & 3.3 \\
\hline$\theta_{m}$ & -30.0 & $o_{m}$ & -30.0 \\
\hline$\theta_{h}$ & -39.0 & $o_{h}$ & -39.0 \\
\hline$\theta_{n}$ & -32.0 & $o_{n}$ & -32.0 \\
\hline$\theta_{r}$ & -67.0 & $o_{r}$ & -67.0 \\
\hline$\theta_{a}$ & -63.0 & $o_{a}$ & -63.0 \\
\hline$\theta_{b}$ & 0.4 & $o_{b}$ & 0.4 \\
\hline$\theta_{s}$ & -39.0 & $o_{S}$ & 8.0 \\
\hline$\mu_{h}$ & -57.0 & $\gamma_{h}$ & -3.0 \\
\hline$\mu_{n}$ & -80.0 & $\gamma_{n}$ & -26.0 \\
\hline$\mu_{r}$ & 68.0 & $\gamma_{r}$ & -2.2 \\
\hline$k_{1}$ & 15.0 & $k_{\mathrm{Ca}}$ & 22.5 \\
\hline$\Gamma$ & $0.0375 \frac{\mathrm{mol}}{\left(\mid \mathrm{As}^{2}\right)}$ & $c_{\mathrm{m}}$ & $1 \frac{\mathrm{pF}}{\mu m^{2}}$ \\
\hline
\end{tabular}
parameters for the reversal potentials and maximum conductances of the respective ion channels were Gaussian distributed around the mean values given in Tables $\mathbf{1}, \mathbf{2}$. We chose a standard deviation equal to $5 \%$ of the mean value. Thus, not all neurons were identical, which led to a more realistic scenario. A schematic diagram of our model network is illustrated in Figure 1. We
Table 1 | Parameter values used for STN neurons.

The maximum conductances $g_{i o n}$ and reversal potentials $v_{\text {ion }}$ were Gaussian distributed with a standard deviation of $5 \%$ around the respective mean values given in this table.

focussed on a detailed description of the connections within and between the two nuclei, the STN and the GPe. Hence, our model consists of two mutually interacting sub-networks representing the STN and the GPe. The connectivity matrix of the entire network could be written as a combination of several sub-networks:

$$
W=\left(\begin{array}{ll}
w_{\mathrm{ss}} & w_{\mathrm{gs}} \\
w_{\mathrm{sg}} & w_{\mathrm{gg}}
\end{array}\right)
$$

$w_{\mathrm{ss}}, w_{\mathrm{gs}}, w_{\mathrm{sg}}$, and $w_{\mathrm{gg}}$ represent the connection matrices for the STN-STN (ss), STN-GPe (sg), GPe-STN (gs), and GPe-GPe (gg) sub-networks, respectively. Since we considered the simulated neuronal network as a weighted and directed graph, the matrix was not necessarily symmetric and each entry $W_{i j}$ corresponded to the connection strength between neuron $i$ and $j$. We excluded self-connections in our model, which resulted in: $W_{i i}=0 \forall_{i}$. 
Table 2 | Parameter values used for GPe neurons.

\begin{tabular}{|c|c|c|c|}
\hline & $\mathrm{nS} / \mu \mathbf{m}^{2}$ & & $\mathbf{m V}$ \\
\hline$g\llcorner$ & 0.1 & $v_{\mathrm{L}}$ & -55.0 \\
\hline$g_{\mathrm{K}}$ & 30.0 & $v_{K}$ & -80.0 \\
\hline$g_{\mathrm{Na}}$ & 120.0 & $v_{\mathrm{Na}}$ & 55.0 \\
\hline$g_{\mathrm{Ca}}$ & 0.15 & $v_{\mathrm{Ca}}$ & 120.0 \\
\hline$g_{\mathrm{ahp}}$ & 30.0 & $v_{\mathrm{gg}}$ & -80.0 \\
\hline \multirow[t]{2}{*}{$g_{\top}$} & 0.5 & $v_{\mathrm{gs}}$ & -100.0 \\
\hline & $\mathrm{ms}$ & & ms \\
\hline$\eta_{h}$ & 0.27 & $\xi_{h}$ & 0.05 \\
\hline$\eta_{n}$ & 0.27 & $\xi_{n}$ & 0.05 \\
\hline$\eta_{r}$ & 30.0 & & \\
\hline$\vartheta_{h}$ & 0.05 & $\vartheta_{n}$ & 0.05 \\
\hline$\vartheta_{r}$ & 1.0 & & \\
\hline$\tau_{\mathrm{gg}}$ & 3.3 & $\tau_{\mathrm{sg}}$ & 1.0 \\
\hline$\theta_{m}$ & -37.0 & $o_{m}$ & 10.0 \\
\hline$\theta_{h}$ & -58.0 & $o_{h}$ & -12.0 \\
\hline$\theta_{n}$ & -50.0 & $o_{n}$ & 14.0 \\
\hline$\theta_{r}$ & -70.0 & $o_{r}$ & -2.0 \\
\hline$\theta_{a}$ & -57.0 & $o_{a}$ & 2.0 \\
\hline$\theta_{s}$ & -35.0 & $o_{s}$ & 2.0 \\
\hline$\mu_{h}$ & -40.0 & $\gamma_{h}$ & -12.0 \\
\hline$\mu_{n}$ & -40.0 & $\gamma_{n}$ & -12.0 \\
\hline$k_{1}$ & 30.0 & $k_{\mathrm{Ca}}$ & 20.0 \\
\hline$\Gamma$ & $0.1 \frac{\mathrm{mol}}{\left(\mid \mathrm{AAs} \mathrm{s}^{2}\right)}$ & $c_{\mathrm{m}}$ & $1 \frac{\mathrm{pF}}{\mu m^{2}}$ \\
\hline
\end{tabular}

The maximum conductances gion and reversal potentials vion were Gaussian distributed with a standard deviation of $5 \%$ around the respective mean values given in this table.

\subsection{THREE-DIMENSIONAL MODEL OF THE STN AND THE GPe}

In our network we modeled $10^{4}$ neurons each within the STN and the GPe. We had to make a trade-off between a physiologically realistic number of neurons in our system and the required computation time. Because the GPe fills a larger volume and has a lower density of neurons than the STN, it was reasonable to choose the same number of neurons for both nuclei (Levesque and Parent, 2005). In order to incorporate anatomical constraints conceding the spatial location of the STN and the GPe into our model, we made use of magnetic resonance imaging (MRI) data taken from a PD patient before DBS surgery. Both nuclei of the left brain hemisphere were segmented on slices of the MRI data by an experienced neurosurgeon. The segmentation contours are illustrated in green in Figure 2. In a second step we manually fitted an ellipsoid [given by $(x / a)^{2}+$ $(y / b)^{2}+(z / c)^{2}-1 \leq 0$ ] into each of the obtained segmentation contours in order to approximate the anatomical shape of both structures. These two ellipsoid structures were filled with uniformly randomly distributed pointlike neurons. In this way we created a spatial model of the STN and the GPe, which is illustrated in Figure 2. The approximated values of the semi-principal axes for the two ellipsoids were: $a_{\text {stn }}=2.5 \mathrm{~mm}, b_{\text {stn }}=6.0 \mathrm{~mm}$,
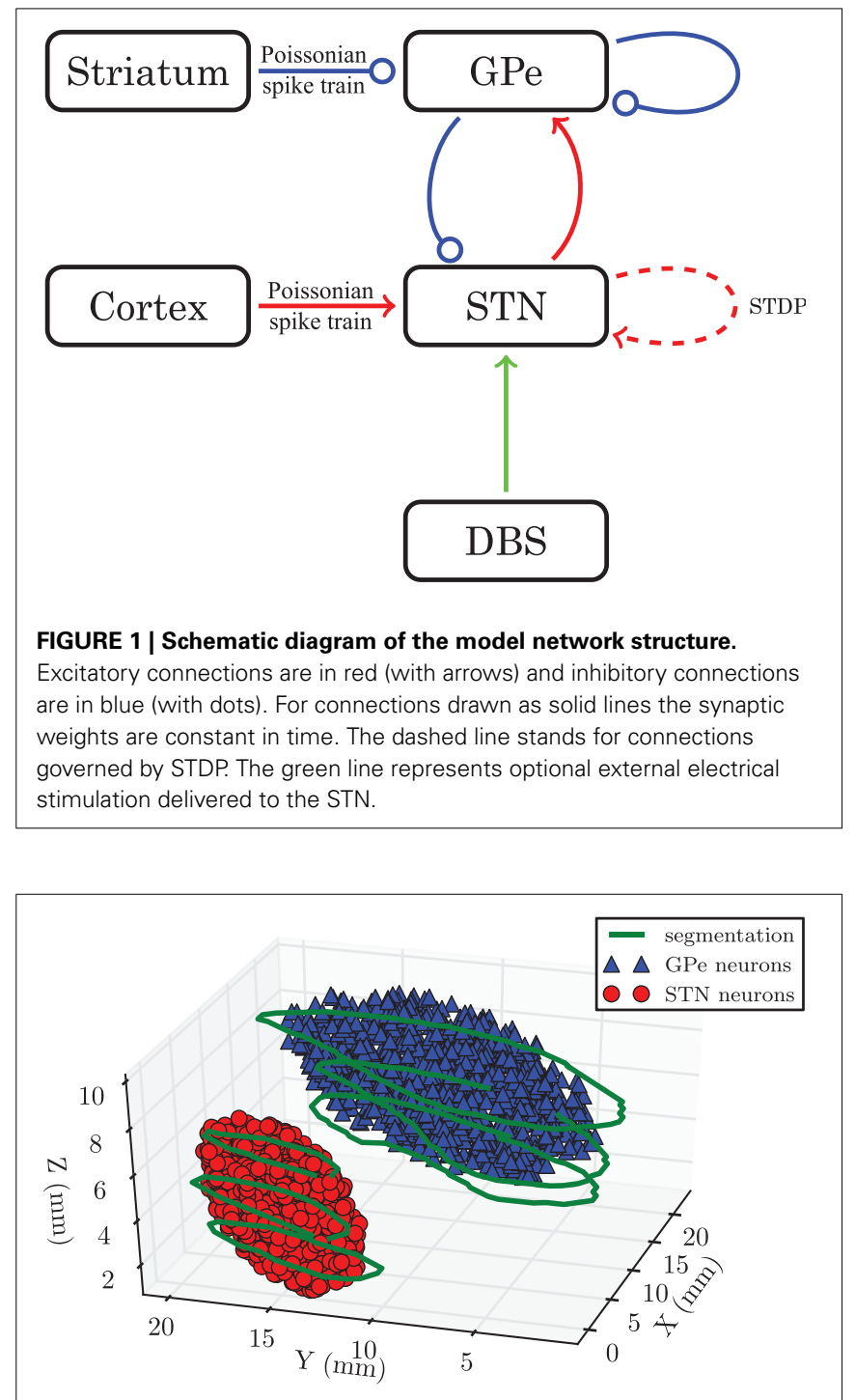

FIGURE 2 | Spatial model of STN and GPe being part of the left brain hemisphere. Contours (shown in green) were segmented on MRI slices taken from a PD patient prior to DBS surgery. We approximated the spatial structure of the STN and the GPe with fits of two ellipsoids. Each of these volumes was filled with $10^{4}$ randomly distributed pointlike neurons.

$c_{\mathrm{stn}}=3.0 \mathrm{~mm}$ for the STN, and $a_{\mathrm{gpe}}=4.6 \mathrm{~mm}, b_{\mathrm{gpe}}=12.3 \mathrm{~mm}$, $c_{\text {gpe }}=3.2 \mathrm{~mm}$ for the GPe. Therefore, the resulting STN volume was $V_{\text {stn }}=188.5 \mathrm{~mm}^{3}$, which is in good accordance to a mean volume of $174.5 \pm 20.4 \mathrm{~mm}^{3}$ reported in the literature (Levesque and Parent, 2005). The GPe volume is not presented here, because it is not the stimulation target and, thus, in the framework of our simulations the geometric dimensions are not crucial.

Although it is still up for discussion whether synaptic connections between STN neurons exist, there has been substantial experimental and theoretical evidence, which supports our assumption of the existence of excitatory connections among STN neurons (Gillies and Willshaw, 1998, 2004; Gillies et al., 2002; Shen et al., 2003; Shen and Johnson, 2006; Baufreton and Bevan, 2008). We based our connectivity value on a theoretical 
estimation given in Gillies and Willshaw (1998). Hence, each STN neuron extended connections to $p_{\mathrm{ss}}=7 \%$ of the entire STN population, resulting in 700 synapses per neuron and $7 \cdot 10^{6}$ synapses in total. The synaptic delays for all connections within the STN were extrapolated from values for gg connections reported in Holgado et al. (2010) and set to a homogeneous value of $\delta_{\mathrm{ss}}=$ $4.0 \mathrm{~ms}$. According to Holgado et al. (2010) we assumed that the corresponding time delays within both structures are shorter than the delays between GPe and STN. The connection probability between STN neurons declined exponentially with increasing distance (see Figure 3 ) and was given by $p(x)=e^{-x / c_{d}}$. The basis for our proposed distance dependence was the extent of the dendritic tree surrounding a STN neuron. In accordance with (Hellwig, 2000) we assumed that a synaptic contact is present when an axonal and a dendritic branch are spatially close to each other. Because the density of dendritic branches is lower for larger distances from the soma, the probability of a touch (and thus a synapse) between a dendritic tree and an axon decreases with increasing distance (Hellwig, 2000). The range of the dendrites varies from 0.2 to $0.9 \mathrm{~mm}$ between individual STN neurons and has a mean of $\bar{r}_{d}=0.543 \mathrm{~mm}$ (Kita et al., 1983; Afsharpour, 1985). We adjusted the decay constant $c_{d}=0.5$ such that the connection probability for the mean range of the dendritic tree $\bar{r}_{d}$ was still $33 \%$ and the probability for connections above the theoretical maximum distance of $1.9 \mathrm{~mm}$ was considerably low. While we chose a distance dependent probability for connections between neurons, the initial synaptic weights of the connections were Gaussian distributed around a mean value $\bar{w}_{\mathrm{ss}}(0)$ with standard deviation $\sigma_{\text {ss }}$ without any distance dependence.

In contrast to the STN, the connectivity within the GPe was significantly lower with each neuron contacting only $1 \%$ of the entire population (Sadek et al., 2007), resulting in 100 synapses per neuron and $10^{6}$ synapses in total for our model. Connections between GPe neurons were inhibitory. We chose the same distance dependence as reported above, but adjusted the parameter $c_{d}=0.63$ in accordance to the increased reach of the dendritic tree, which ranged to a maximum of $1.5 \mathrm{~mm}$ with a mean of $\bar{r}_{\mathrm{d}}=$ $0.7 \mathrm{~mm}$ (Yelnik et al., 1984; Sadek et al., 2007). For the synaptic delay we chose $\delta_{\mathrm{ss}}=4.0 \mathrm{~ms}$ as suggested in Holgado et al. (2010).

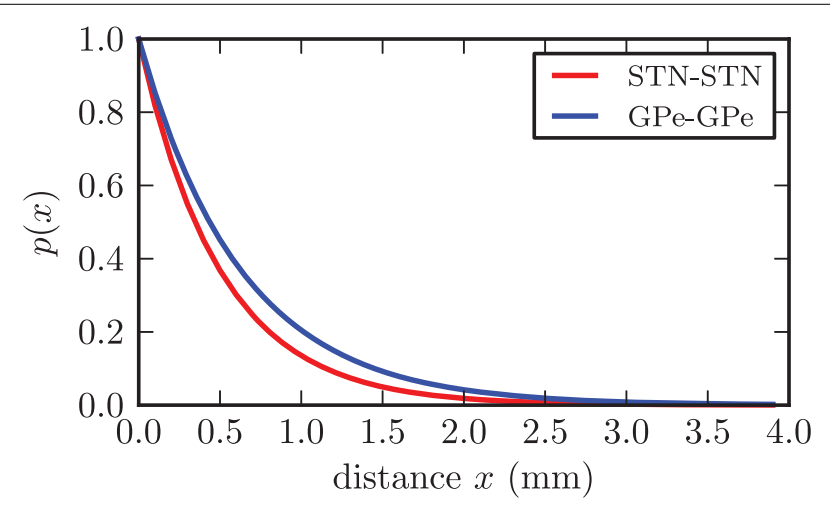

FIGURE 3 | Illustration of the dependence between connection probability and distance between two neurons. Both functions fulfilled the constraint $p\left(\bar{r}_{\mathrm{d}}\right)=0.33$.
Similar to the connections within the STN, the synaptic weights were Gaussian distributed with a mean value of $\bar{w}_{\mathrm{gg}}=0.25 \cdot 10^{-3}$ and standard deviation $\sigma_{\mathrm{gg}}=0.125 \cdot 10^{-3}$.

Besides the connections within the two simulated parts of the basal ganglia, both structures are also tightly interconnected (Shink et al., 1996). First, the STN has an excitatory influence on the GPe and on the other hand the GPe exerts an inhibitory impact on the STN neurons. These long range connections are established by axons extending from the STN to the GPe and vice versa. Therefore, we did not use distance dependent connection probabilities for links between STN and GPe, but we relied only on the connectivity values of $2 \%$ reported in Baufreton et al. (2009). This resulted in a fixed number of 200 synapses per neuron, contacting randomly chosen targets in the distant nucleus, respectively. The transmission delay of $\delta_{\mathrm{sg}}=\delta_{\mathrm{gs}}=$ $4.0 \mathrm{~ms}$ for connections between the STN and the GPe is published in Fujimoto and Kita (1993) and Kita et al. (2005). Likewise to the above described connections, the initial values for the synaptic weight were Gaussian distributed around the mean values $\bar{w}_{\mathrm{sg}}=6.0 \cdot 10^{-3}$ and $\bar{w}_{\mathrm{gs}}=3.0 \cdot 10^{-3}$ with respective standard deviations $\sigma_{\mathrm{sg}}=0.3 \cdot 10^{-3}$ and $\sigma_{\mathrm{gs}}=0.15 \cdot 10^{-3}$.

\subsection{SYNAPTIC CURRENTS}

In our model each incoming spike triggers a postsynaptic current with the shape of an $\alpha$-function (Dayan and Abbott, 2000; Gerstner and Kistler, 2002). The postsynaptic current generated by a spike at time $t_{k}$ reads:

$$
\alpha(t)=\frac{t-t_{k}}{\tau_{\mathrm{syn}}^{2}} e^{-\left(t-t_{k}\right) / \tau_{\mathrm{syn}}}, t_{k} \leq t<t_{k+1}
$$

This yields the following coupling term, which quantifies the total synaptic input current to a postsynaptic neuron $i$ received from presynaptic neurons $j$ :

$$
I_{\text {syn }}(t)=\sum_{j} W_{i j}\left(v_{i}(t)-v_{\text {syn }}\right) \alpha(t)
$$

$W_{i j}$ denotes the synaptic weight (coupling strength) between presynaptic neurons $j$ and the postsynaptic neuron $i$. $v_{\text {syn }}$ is the reversal potential, at which a given neurotransmitter causes no net current flow of ions through the ion channels associated with that particular neurotransmitter receptor. Therefore, $v_{\text {syn }}$ depends on the types of connected neurons and on whether the connection is excitatory or inhibitory. In our model, there exist four types of synapses for ss, sg, gs, and gg connections. The exact values of $v_{\text {syn }}$ and $\tau_{\text {syn }}$ for the four different types of connections within our model are given in Tables 1, 2.

\subsubsection{Noise inputs}

The external inhibitory input from the striatum to the GPe is modeled by a constant negative input current of $I_{\text {app }}=-7.0 \mathrm{pA}$ applied to all GPe neurons and additional Poissonian spike trains with a frequency of $f_{\mathrm{p}}^{\mathrm{gpe}}=40 \mathrm{~Hz}$.

$$
I_{\text {noise }}=\sum_{j=0}^{P_{\lambda}(i)} w_{\text {noise }}\left(v_{i}(t)-v_{\text {noise }}\right) \alpha(t),
$$


with the Poisson distribution $P_{\lambda}(i)$ and $\lambda=f_{\mathrm{p}} \delta$ with the observation (simulated) time interval of the Poisson distribution $\delta$ between subsequent time steps, which is equal to the time resolution of the simulation. Likewise, we mimick the excitatory connections from the cortex to the STN also by feeding Poissonian distributed spikes with a frequency of $f_{\mathrm{p}}^{\text {stn }}=20 \mathrm{~Hz}$ into each STN neuron. We choose $f_{\mathrm{p}}^{\text {gpe }}=2 \cdot f_{\mathrm{p}}^{\text {stn }}$, so that the physiological mean firing rate in the GPe is approximately twice the mean firing rate of the STN, as reported from experiments (Steigerwald et al., 2008; Nishibayashi et al., 2011; Wichmann et al., 2011). We do not feed the same spike train to all neurons, but each neuron receives a unique Poissonian spike train. For both the STN and the GPe the connection strength for the noise input is $w_{\text {noise }}=0.2$, the value of the time constants for the $\alpha$-function is $\tau_{\text {noise }}=$ $1.0 \mathrm{~ms}$, and the reversal potential is chosen to be equal to $v_{\text {noise }}=0 \mathrm{mV}$.

\subsection{SYNAPTIC PLASTICITY}

We consider the synaptic strength of connections between STN neurons to be dynamical variables. Therefore, we introduce a learning rule that included synaptic weights, which are dependent on the timing of the firing of pre- and postsynaptic STN neurons (Gerstner et al., 1996; Markram et al., 1997; Bi and Poo, 2001). Our hypothesis that STN connections are governed by STDP is based on the findings reported in Shen et al. (2003), where longterm depression (LTD) and potentiation (LTP) could be induced by stimulation in rat STN neurons.

We use an additive spike timing-dependent plasticity (STDP) rule, which is illustrated in Figure $\mathbf{4 A}$ and defined by the following equation:

$$
\Delta w_{i j}(\Delta t)=\left\{\begin{array}{cc}
\lambda e^{-\frac{|\Delta t|}{\tau_{+}}} & \Delta t>0, \\
-\lambda \beta e^{-\frac{|\Delta t|}{\tau_{-}}} \quad \Delta t \leq 0
\end{array}\right.
$$

In a previous study it has been shown that in a Kuramoto model of coupled phase oscillators multistability only occurs for asymmetric STDP rules (Maistrenko et al., 2007). This asymmetry was substantiated experimentally in different brain regions (e.g., hippocampus Bi and Poo, 1998 and neocortex Feldman, 2000). Therefore, we also used an asymmetric STDP rule with the following parameters: $\tau_{-}=27.5, \tau_{+}=12.0, \lambda=2.0 \cdot 10^{-3}$, and $\beta=1.1 . \tau_{-}$and $\tau_{+}$are the time constants for the synaptic weight
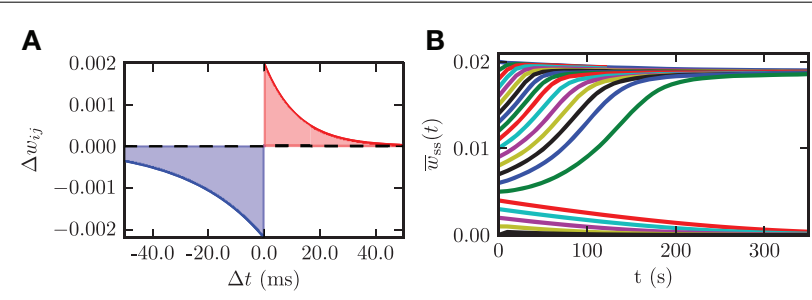

FIGURE 4 | (A) STDP rule used for the connections among STN neurons in the model network. Depression is shown in blue and potentiation is shown in red. (B) Time evolution of the mean synaptic weight among STN neurons $\bar{W}_{\mathrm{ss}}(t)$ for different initial values $\bar{w}_{\mathrm{ss}}(0)$. change of depression and potentiation, respectively. $\lambda$ quantifies the impact of a single spike on the synaptic weight change and can be considered as the learning rate of the synaptic connection. The parameter $\beta$ specifies the ratio between depression and potentiation in the synaptic learning rule. The synaptic weights among STN neurons are confined to the interval $w_{i j}^{\mathrm{ss}}(t) \in\left[0, w_{\max }^{\mathrm{ss}}\right]$, where $w_{\max }^{\text {ss }}=20 \cdot 10^{-3}$. This upper boundary prohibits an unphysiological non-saturated increase in synaptic weights. We use hard bounds for the upper and lower boundary in our simulations. We use the same parameters for all synaptic connections among STN neurons. In order to investigate the dynamics of the model system with plasticity involved, we varied the initial mean synaptic weight among STN neurons $\bar{w}_{\mathrm{sS}}(0)$ and observed the time course of the mean synaptic weight $\bar{w}_{\text {ss }}(t)$. The initial values for the mean coupling were Gaussian distributed with a standard deviation of $5 \%$ of the maximum weight $\sigma_{\mathrm{ss}}=0.1 \cdot 10^{-3}$. These simulations show that the system approaches one of the two dominating attractors with strongly synchronized activity (high mean coupling strength) or strongly desynchronized activity (low mean coupling strength). Below a certain threshold value in the initial mean synaptic weight $\bar{w}_{\mathrm{ss}}(0)=5.0 \cdot 10^{-3}$ the system monotonically approaches the desynchronized state, and with an initial value above the threshold the system monotonically converges to the strongly synchronized state (cf. Figure 4B). The plot shows only the time course of the mean synaptic weight among STN neurons within the first $350 \mathrm{~s}$ of neuronal activity. Nevertheless, we simulated the network activity for $600 \mathrm{~s}$, in order to exclude transient or meta-stable behavior. Although STDP might induce many different multistable states, only the strongly desynchronized and strongly synchronized states predominate, and the system ultimately approaches either one of them. For instance, a strong initial connection strength among STN neurons leads to an inception of synchronized activity. This causes the neurons to fire nearly simultaneously, and this narrow spike timing results in a potentiation of the coupling strength and thus stabilizes the strong coupling and associated synchronization. In contrast, weak initial coupling is associated with desynchronized dynamics within the system and therefore results in a further decrease in synaptic connection strength. Hence, the desynchronized and synchronized dynamics are stable in the model system governed by plasticity. STDP facilitates the stabilization of synchronized and desynchronized activity and thus leads to multistability in the STN population. This observation is in good accordance with results from previous studies (Tass and Majtanik, 2006; Hauptmann and Tass, 2007; Popovych and Tass, 2012). The effects described above are robust against variations in the standard deviation of the initial mean coupling strength of up to $20 \%$ of the maximum weight.

\subsubsection{Stimulation input}

For DBS the electrical stimulation is administered to the neuronal tissue in form of short biphasic current or voltage pulses (Cogan, 2008). A typical current pulse $P(t)$ for stimulation of neuronal tissue has a cathodal and an anodal phase with current amplitudes and durations that result in an overall zero net charge for the entire pulse (to guarantee chargebalance): 


$$
P(t)=\left\{\begin{array}{cl}
\kappa & t_{k} \leq t<t_{k}+\omega \\
-\kappa / p_{s} & t_{k}+\omega \leq t<t_{k}+\omega\left(1+p_{s}\right) \\
0 & \text { else }
\end{array}\right.
$$

where $t_{k}$ are the onset times of the current pulses, $\kappa$ is the amplitude, and $\omega$ is the width of the cathodal pulse. $p_{s}$ determines the duration and amplitude of the charge-balancing anodal pulse part. This charge-balance is important in order to avoid permanent charge transfer into the neuronal tissue, which might cause permanent tissue damage. For details of the possible electrochemical processes occurring at the electrode-tissue interface we refer to Cogan (2008).

The spatial arrangement of the stimulated neurons and the stimulation sites is crucial if the impact of electrical stimulation on a neuronal population is investigated. The influence of electrical stimulation on an individual neuron strongly depends on the relative positions within the three-dimensional coordinate system. One of the clinically most commonly used electrodes for DBS is the Medtronic DBS lead model 3389. The distal end with four electrode contacts is inserted into the target area. The electrode consists of four separate cylindrical contacts made of a Pt-Ir alloy with a typical length of $1.5 \mathrm{~mm}$ (Coffey, 2009). The electric field of such a cylindrical electrode is approximately radial and decreases inversely proportional to the distance. We used an analytic approximation based on the electric field of a line charge of finite length in order to estimate the distance dependence of the stimulation strength (Richardson et al., 2003). Furthermore, we assumed a homogeneous resistivity of $1.25 \Omega \mathrm{m}$ for the surrounding neuronal tissue. The approximation reads (Richardson et al., 2003):

$$
S\left(d_{i j}\right)=\frac{1}{d_{i j} l_{c} \sqrt{1+4\left(d_{i j} / l_{c}\right)^{2}}}
$$

The function $S\left(d_{i j}\right)$ models the distance dependent decay of stimulation strength with $d_{i j}$ representing the distance between neuron $i$ and the location of the stimulation contact $j$. Additionally, $S\left(d_{i j}\right)$ depends on the length of the electrode contacts $l_{c}$.

In order to eliminate the singularity at $d_{i j}=0$, we assume a minimal distance of $d_{\text {min }}=0.7 \mathrm{~mm}$ to the electrode for all neurons. We achieve this by prohibiting possible neuron coordinates within a cylindrical volume with $1.4 \mathrm{~mm}$ diameter surrounding the electrode axis. This electrode canal is slightly larger than the electrode diameter and is created during the implantation surgery.

We apply CR stimulation (Tass, 2003a,c) to the modeled network of STN neurons. Each neuron receives stimulation input from all $M$ stimulation contacts. The distance dependence of the stimulation strength causes the formation of $M$ different sub-populations, because only the neurons close to a particular stimulation site are influenced sufficiently in order to alter their firing behavior. According to the CR stimulation protocol $M$ different sub-populations are stimulated via $M$ different stimulation sites at equidistant timing points by application of short bursts of current pulses within a stimulation period $T$, which approximately matches the period of the targeted pathological oscillation.
$T_{p}$ denotes the stimulation period within a burst. For our simulations we chose $M=4$, which matches the number of available electrode contacts for the considered electrode type Medtronic 3389. In contrast to the sequential CR algorithm, we randomized the order of activated contacts between stimulation periods (i.e., stimulation cycles) (Figure 5). The only constraint was that the same contact could not be activated twice in a row between subsequent cycles. This modification of the CR stimulation protocol has previously been successfully used in an animal experiment with Parkinsonian monkeys (Tass et al., 2012b). Three cycles of $\mathrm{CR}$ stimulation (ON-cycles) were followed by two cycles without stimulation (OFF-cycles). According to a previous computational study this alternating pattern of 3 stimulation ON- and 2 stimulation OFF-cycles is superior to permanent CR DBS (without OFF-cycles), since during the OFF-cycles the CR-induced desynchronization is maximal (Lysyansky et al., 2011). Throughout our simulations we only varied the stimulation amplitude $\kappa$. The remaining stimulation parameters were fixed to $T_{p}=7.69 \mathrm{~ms}$, $\omega=200 \mu \mathrm{s}, p_{s}=8$, and $T=125.0 \mathrm{~ms}$.

Therefore, the effective stimulation signal received by neuron $i$ from stimulation contact $j$ is given by multiplication of the stimulation amplitude with the spatial profile defined by Equation 15 and reads:

$$
I_{\text {stim }}(t)=\sum_{j=1}^{M} S\left(d_{i j}\right) \rho_{j}(t) P(t)
$$

The function $\rho_{j}(t)$ is an indicator function, which controls the activation of the respective stimulation site $j . M$ is the number of activated stimulation contacts, where $M=4$ for a typical electrode.

In our model we inject the stimulation current exclusively to the soma of the stimulated cells. Thus, our model omits various proposed effects of DBS (e.g., axonal activation). However, a recent computational study showed, that DBS of local STN cells located within the nucleus is more beneficial than the stimulation of fibers of passage (So et al., 2012). Nevertheless, our investigations revealed that the observed mixture of firing

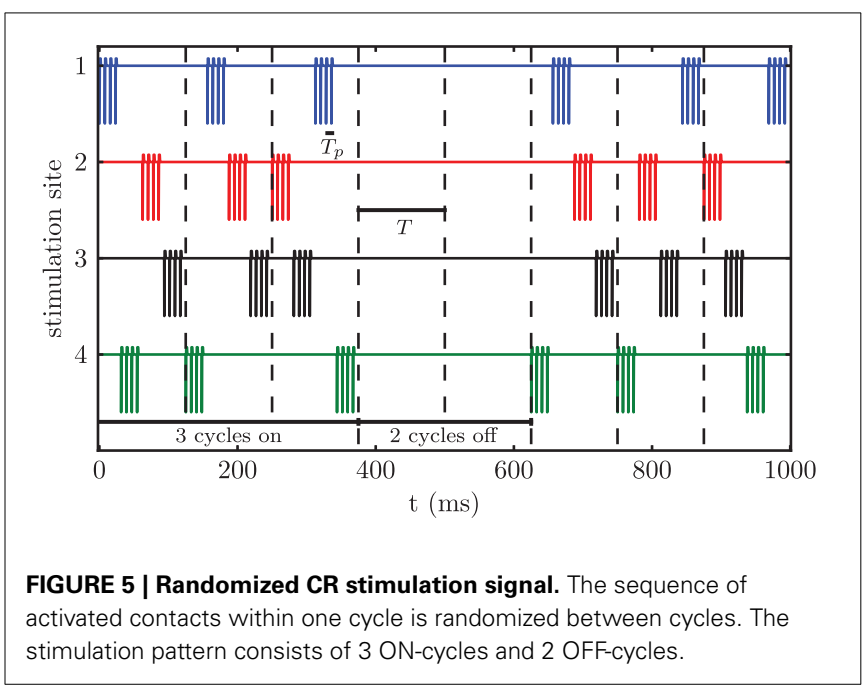


rates within the STN population is consistent with the interplay of effects reported or predicted for HF stimulation (Grill and McIntyre, 2001; McIntyre et al., 2004a; Miocinovic et al., 2006; Hauptmann and Tass, 2007). While some STN neurons showed stimulus-locked firing, mimicking the stimulus-locked somatic or axonal generation of action potentials in STN neurons, other STN neurons were completely or partially inhibited. Similar to Humphries and Gurney (2012) this mixture entirely arose from the interaction between the stimulation pulses fed into STN neurons and the effects induced by the reciprocal connections within the simulated STN-GPe network. This effect has also been reported previously for other simplified models of electrical stimulation (Humphries and Gurney, 2012). However, it is unquestioned that the mechanism of action of DBS includes suppression of synaptic input and direct (including retrograde) activation of axons (Ranck, 1975; Grill and McIntyre, 2001; McIntyre et al., 2004a; Miocinovic et al., 2006; Hauptmann and Tass, 2007; Gradinaru et al., 2009).

\subsection{SIMULATION OBSERVABLES}

Previous studies (Kuramoto, 1984; Daido, 1992; Tass, 1999) have shown that the phenomenon of synchronization within a neuronal model system can be quantified by the order parameter $R(t)$. The order parameter $R(t)$ bases on the phases $\phi_{j}(t)$ of the individual neurons of the neuronal model system and is defined by:

$$
R(t) e^{i \psi(t)}=\frac{1}{n} \sum_{j=1}^{n} e^{i \phi_{j}(t)}
$$

$n$ denotes the number of neurons contained in the system. $\psi(t)$ is the mean phase of the neuronal system and the quantity $\phi_{j}(t)$ represents the phase of each individual neuron $j$, which is defined by linear interpolation between two subsequent spikes at times $t_{j, k}$ and $t_{j, k+1}(k=0,1,2, \ldots)$. Thus, the phase of a neuron ranges in the interval $[0,2 \pi$ ) and is given by the following equation (Pinsky and Rinzel, 1995):

$$
\phi_{j}(t)=\frac{2 \pi\left(t-t_{j, k}\right)}{t_{j, k+1}-t_{j, k}}, \quad t_{j, k} \leq t<t_{j, k+1}
$$

The above definition of a neuronal phase requires an adequate spike detection. We detected spikes by a combined threshold and local maximum search: If there was a local maximum above a certain threshold of the membrane potential, it was considered a spike. We performed the spike detection with an accuracy of $0.1 \mathrm{~ms}$, which was sufficient according to a typical spike width at half-maximal spike amplitude of approximately $2.0 \mathrm{~ms}$. We did not distinguish between single spikes and bursts (episodes of rapid spiking). $R(t)$ quantifies the coherence of the phases and is ranging from 0 to 1 , where $R(t)=1$ corresponds to a fully synchronized system, while values close to zero correspond to complete absence of phase synchronization. Due to finite size fluctuations $R(t)$ never reaches zero, $\frac{1}{\sqrt{n}}$ is the expected value. We measured the degree of synchronization separately for the STN and GPe sub-networks. For example, in the desynchronized regime the time course of the first order parameter $R(t)$ is stable and close to zero in both neuronal populations with time averages of $\langle R\rangle_{\text {stn }}=0.009$ and $\langle R\rangle_{\text {gpe }}=0.009$, which are in good accordance with the expected value of $R(t)=\frac{1}{\sqrt{n}}=0.01$. In contrast, in the synchronized regime $R(t)$ is stable and close to 1 for the STN sub-network with an average value of $\left\langle R_{1}\right\rangle_{\text {stn }}=0.97$ indicating almost perfect in-phase synchronization. For the GPe population the values of the order parameter are considerably lower with a time average of $\left\langle R_{1}\right\rangle_{\text {gpe }}=0.67$. The weakly coupled GPe neurons are periodically synchronized by the external force of the STN. In order to smooth out short-term fluctuations and highlight long-term trends, we calculated a moving average over the recorded data. Thereby we used a time window of $1000 \mathrm{~ms}$ duration.

In order to predict an optimal location for the DBS electrode we additionally studied the spatial distribution of synchronization within the STN volume. Therefore, we introduced a method to measure local synchronization within the STN, based on the order parameter described above. In our approach we subdivided the volume of the STN in equally sized cubic voxels and computed the order parameter $R_{k}(t)$ for the set of $N_{k}$ neurons contained within each voxel $k$ separately.

$$
R_{k}(t) e^{i \psi_{k}(t)}=\frac{1}{N_{k}} \sum_{j=1}^{N_{k}} e^{i \phi_{j}(t)}
$$

Synchrony was calculated for each voxel individually. The voxels do not overlap. The high density of neurons in our large-scale model guarantees that each voxel contains approximately $\overline{N_{k}} \approx$ 42 neurons. Nevertheless, we only considered voxels containing at least 10 neurons. For voxels with a low number of contained neurons the distinction between partly and fully synchronized states can become difficult due to the finite size fluctuations mentioned above. This also limits the spatial resolution of this synchrony measure. In order to estimate the homogeneity of local synchronization within the STN volume, we define the following local order parameter:

$$
\bar{r}_{1}=\frac{1}{N_{\mathrm{V}}} \sum_{k=1}^{N_{\mathrm{V}}}\left\langle R_{k}\right\rangle
$$

$\bar{r}_{1}$ is close to one if the entire STN volume is strongly synchronized and close to zero if it is strongly desynchronized. $N_{\mathrm{V}}=232$ denotes the number of voxels considered for the calculation. Note, $\bar{r}_{1}$ is close to one if either the whole STN population is synchronized and constitutes a giant oscillator, or if the STN population is synchronized on the level of single voxels only, whereas between different voxels there might be non-vanishing phase shifts. The standard deviation of this local order parameter $\sigma_{\bar{r}_{1}}$ incorporated additional information about the homogeneity of the local synchronization. Large values of $\sigma_{\bar{r}_{1}}$ indicate high discrepancies in the local order parameter between different regions of the STN volume.

\section{RESULTS \\ 3.1. SCALABILITY TEST}

The large number of simulated neurons made it necessary to parallelize the numerical computations. In our performance tests, we 
focused on the strong scaling of our model. Strong scaling characterizes the ability of an application to perform the same sized calculation faster on computers with more processors (Plesser et al., 2007). The network was built according to the scheme described above. Thus, the resulting network contained $2 \cdot 10^{4}$ nodes connected via $1.2 \cdot 10^{7}$ synapses. Only the synaptic weights between the STN neurons were governed by a STDP rule, while all remaining connections were constant in time. We focused on the simulation of the network activity and omitted the online data analysis in the scalability tests. The time evolution of this network was simulated for one biological second and the runtime was measured. To test the strong scaling behavior of the simulation program, the problem size was fixed and the number of used processors on the supercomputer was increased from 8 to 2048 processors. During simulations the neurons of the entire simulated network were active with a mean firing rate of $15.8 \mathrm{~Hz}$. The scalability of our simulation is illustrated in Figure 6. The speedup factor quantifies how much faster the simulation runs on $n$ processors, with runtime $T_{n}$, compared to a simulation on 8 processors, $\left(T_{8}\right)$. Thus, the speed-up factor is given by $S=T_{8} / T_{n}$. For up to 128 used processors our scalability test showed a slightly steeper slope than the ideal linear speed-up (see Figure 6B, i.e., for up to 128 processors the speed-up marks "+" are positioned above the ideal line of linear speed-up). A maximal slope for the speed-up of 1.1 was observed (averaged over 10 independent trials). Although the linear speed-up is commonly accepted to be the theoretical maximum, this supralinear behavior of our simulation can be explained by the fast cache memory increasing proportionally to the number of used processors (Morrison et al., 2005). Thus, the ratio between the locally required working memory and the locally available cache memory decreases, which leads to a performance gain (Wilkinson and Allen, 2004). Note, the supralinear speed-up might be more prominent for simulated networks with higher average firing rates (Morrison et al., 2005). This is because the size of the available cache memory has a stronger impact on the efficiency of writing to the spike buffers for higher firing rates of the simulated neurons.

The computational workload of the individual neurons in a simulated neuronal network has a significant impact on the scaling behavior of the simulation (Plesser et al., 2007). This is due to the fact that a computationally expensive model for individual neurons or a larger number of neurons per processor leads to an increase in the ratio of local computation to communication costs. The overhead due to the communication in a distributed simulation grows with an increasing number of used processors (Plesser et al., 2007). In our performance test this effect balanced the performance gain from efficient caching for 256 processors. The communication overhead became predominant for 2048 processors, which led to a saturation of the gained speedup. In the simulations we performed throughout this work, we typically used 1024 processors. With this setup the simulation of one biological second consumed approximately $3 \mathrm{~s}$ of computation time. This included the required computation time for the online analysis of the simulation results, which was omitted in the scalability tests.

\subsubsection{Spontaneous activity of the model network}

As shown above the system enters one of the two dominant stables states depending on the initial mean synaptic weight among the STN neurons. The observed spontaneous activity within the two states can be characterized in the following way: First, for low mean coupling within the STN the neurons fire in an uncorrelated, desynchronized manner. We consider this type of activity as a model for the non-pathological (healthy) state of the basal ganglia system. Second, the system is capable to fire in a strongly correlated synchronized pattern, if the mean coupling strength within the STN is strong enough. This type of activity models the pathological ("Parkinsonian") state of the STN-GPe network.

Both states can be characterized by multiple observables, e.g., the mean membrane potentials of both populations that have been recorded with $1.0 \mathrm{~ms}$ time resolution. In the desynchronized state the mean membrane potentials of the STN and the GPe population showed only minor fluctuations around the resting value. The time averages over the mean membrane potentials were $\langle\bar{v}(t)\rangle_{\text {stn }}=-59.2 \pm 0.1 \mathrm{mV}$ and $\langle\bar{v}(t)\rangle_{\text {gpe }}=-90.2 \pm 0.1 \mathrm{mV}$.

In order to measure the degree of synchronization within the simulated neuronal network, we therefore calculated the order parameter $R(t)$ (section 2.5), which measured the phase coherence of the neurons and gave us the opportunity to detect possible cluster states. The time course of the order parameter $R(t)$ was stable and close to zero in both neuronal populations with a time average of $\langle R(t)\rangle_{\text {stn }}=0.009$ for the STN and $\langle R(t)\rangle_{\text {gpe }}=0.009$ for the GPe with standard deviations smaller than $10^{-4}$. The time course of the order parameter for both sub-structures was

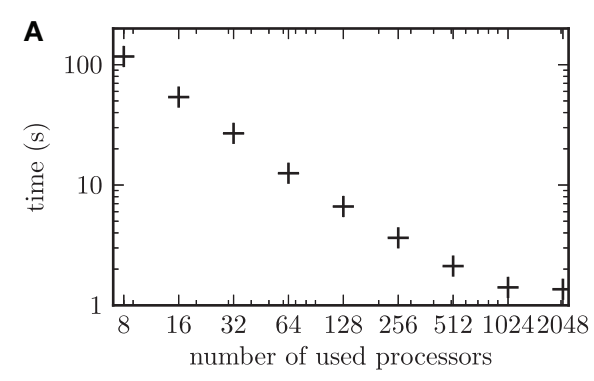

FIGURE 6 | Results of the scalability test. (A) shows the time to simulate 1 biological second of the network activity as a function of the number of used processors in double logarithmic representation. (B)

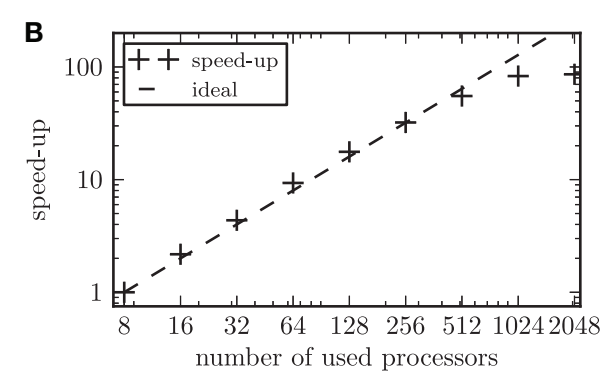

displays the speed-up factor for the simulation times. The symbols $(+)$ represent the measured speedup, while the dashed line marks the ideal speed-up. 
in accordance to what could be expected from a system of noise driven and very weakly coupled oscillators.

An adequate characterization of the observed neuronal activity required the investigation of the firing frequencies of individual neurons and their distribution. Therefore, we calculated the time intervals between subsequent spikes for each neuron separately and thus obtained the distribution of interspike intervals over the two populations of neurons. Hence, this resulted in an interspike interval (ISI) histogram shown in Figure 7. The bin width of the histogram was chosen equal to $5.0 \mathrm{~ms}$. In the desynchronized state the ISI histogram of the STN population showed a broadened distribution of ISIs around the median value of $\tilde{T}_{\text {stn }}=261.2 \mathrm{~ms}$ with an average absolute deviation from the median (MD) of $\mathrm{MD}_{\text {stn }}=$ $51.1 \mathrm{~ms}$. Accordingly, this value corresponded to a median firing rate of $\tilde{f}_{\text {stn }}=3.8 \mathrm{~Hz}$. For the GPe neurons we calculated a median spike period of $\tilde{T}_{\text {gpe }}=92.1 \pm 82.6 \mathrm{~ms}$, which corresponded to a median spike frequency of $\tilde{f}_{\text {gpe }}=10.9 \mathrm{~Hz}$. The ISI distribution for the GPe was asymmetrical and is shown in Figure 7B. The asymmetry occurred due to the fact that the GPe neurons have a tendency to fire short bursts of action potentials. These bursts had relatively short ISIs resulting in a shift of the histogram to ISIs with reduced length. We preferred calculating the median over the mean value, because both distributions were asymmetrical and for a symmetrical distribution mean and median have identical values.

For strong mean coupling among STN neurons $\left(\bar{w}_{\mathrm{SS}}>5.0\right.$. $10^{-3}$ ) the model system showed highly synchronized activity $(R(t) \approx 1)$. The synchronized state can be considered as the pathological, unhealthy state, which mimics the neuronal activity within the basal ganglia system in patients suffering from PD (Lenz et al., 1994; Plenz and Kital, 1999). The STN neurons fired collectively with a median period of $\tilde{T}_{\text {stn }}=122.0 \pm 8.3 \mathrm{~ms}$, which corresponded to a median firing rate of $\tilde{f}=8.2 \mathrm{~Hz}$. Between these episodes of collective firing the individual STN neurons remained almost silent. The GPe neurons were driven by the STN activity and showed collective firing with the same frequency as the STN neurons. Nevertheless, individual GPe neurons emitted action potentials constantly and collective periods of silence were not observed for the GPe population.

The computation of population mean membrane potentials gives similar results. The mean membrane potentials of both populations showed high amplitude oscillations with the same frequency as the synchronized activity of the STN neurons (cf. Figure 8). The synchronized firing of the STN neurons forced periodic collective spike discharges of the GPe neurons. This collective GPe activity led to an increased inhibition of the connected STN cells. Thus, the mean membrane potential of the STN population was strongly hyperpolarized (cf. Figure 8A). This mechanism was the reason for the periods of silence in STN neurons following the episodes of collective firing. However, the inhibition from GPe neurons decayed and the mean membrane potential of the STN population recovered before the next episode of collective firing was launched. The GPe neurons, which were excited by incoming action potentials from collective firing of the STN neurons, reacted to this excitation with the emission of bursts. This bursting activity in the GPe neurons caused the very narrow
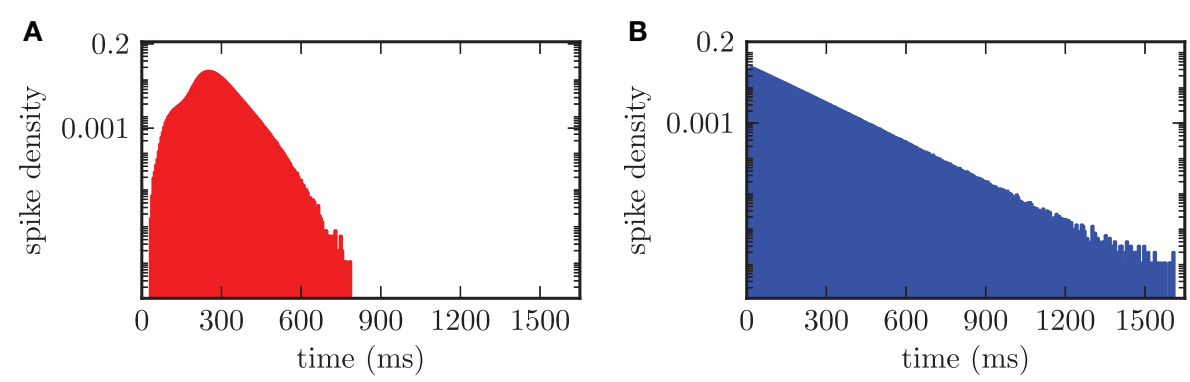

FIGURE 7 | ISI histogram (semi-logarithmic plot) for both neuronal populations in the desynchronized state. (A) Slightly asymmetrical distribution of ISIs for the STN population with a median of $\tilde{T}_{\text {stn }}=261.2 \mathrm{~ms}$. (B) Obvious asymmetry in the ISI histogram, due to bursting activity of the GPe neurons, with $\tilde{T}_{\text {gpe }}=92.1 \mathrm{~ms}$
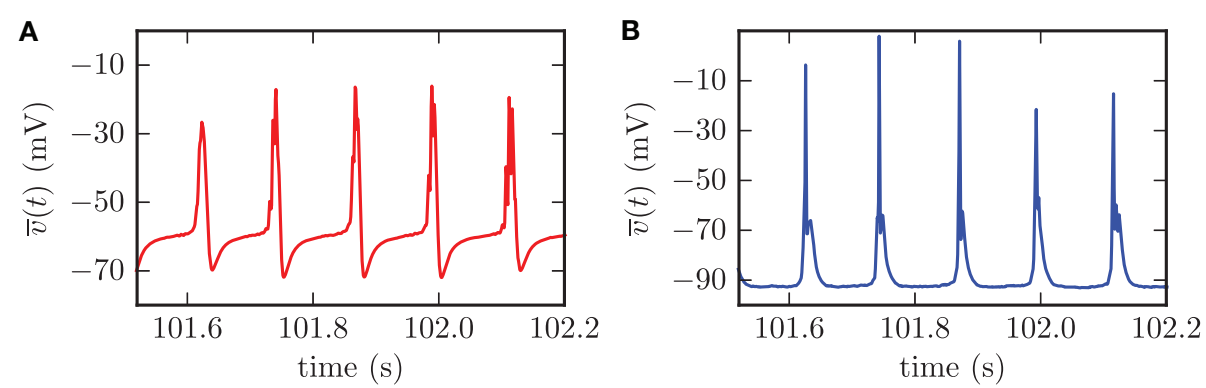

FIGURE 8 | For the synchronized state, the mean membrane potentials of the neurons within the STN (A) and the GPe (B) showed oscillations with large amplitudes. The frequency of these oscillations was the same for both populations. 
peaks in the mean membrane potential of the GPe population (cf. Figure 8B).

A very clear indication of synchronized activity in the simulated network was furthermore provided by the time course of the order parameter $R(t)$. For the STN sub-network $R(t)$ was stable and close to 1 with an average value of $\langle R(t)\rangle_{\text {stn }}=0.97$ indicating almost perfect in-phase synchronization. However, perfect in-phase synchronization was never achieved, because the STN sub-network was composed of non-identical individual neurons and the system always received noise inputs from surrounding structures. For the GPe population the values of the order parameter were considerably lower oscillating around a time average of $\langle R(t)\rangle_{\text {gpe }}=0.67$. This finding can be explained by the fact that the weakly coupled GPe neurons fired in a desynchronized manner most of the time and are periodically synchronized by the external force of the STN. The synchronized input from the STN triggered the collective bursting of the GPe neurons and afterwards the GPe population returned to the desynchronized firing pattern.

The effect of synchronization on the distribution of ISIs is clearly visible in Figure 9. In the synchronized state the median period of spiking activity in STN neurons was shifted toward shorter durations $\tilde{T}_{\text {stn }}=122.0 \pm 8.3 \mathrm{~ms}$. The consequence was an increased frequency $\tilde{f}_{\text {stn }}=8.2 \mathrm{~Hz}$ and much sharper distribution of ISIs (cf. Figure 9A), than for the desynchronized network activity, shown in Figure 7A. The calculation of the median or mean did not make sense for the bi-modal distribution of ISIs in the GPe population shown in Figure 9B. The bi-modal distribution was caused by two reasons. First, the large peak for short time periods around $5-10 \mathrm{~ms}$ could be explained by the increased bursting activity of the GPe neurons. Second, the clearly visible peak at approximately $122.0 \mathrm{~ms}$ was caused by the driving force of connected STN neurons. Additionally, the entire ISI histogram for the GPe population indicated an increased activity distributed over all time periods. This was due to the fact that the frequency of the input from the STN to the GPe was increased in the synchronized regime.

\subsection{OPTIMAL STIMULATION AMPLITUDE}

In all subsequent simulations we set the initial mean synaptic weight among STN neurons to $\bar{w}_{\mathrm{ss}}=(18.0 \pm 0.1) \cdot 10^{-3}$. This corresponded to an initially strongly synchronized activity of the model system. In the first $120 \mathrm{~s}$ of the simulations we left the system unperturbed before we switched on the CR stimulation. As described above, we applied the randomized CR stimulation sequences (Figure 5). The contacts of the stimulation electrode were placed along the $y$-axis of the coordinate system. The $M=4$ electrode contacts were centered within the STN volume with a distance of $2 \mathrm{~mm}$ between two neighboring stimulation sites. In our simulations we only varied the amplitude of the stimulation current pulses $\kappa$ and the location of the electrode contacts. The remaining stimulation parameters were fixed and given by: $\omega=200.0 \mu \mathrm{s}, p_{s}=8.0, T_{p}=7.69 \mathrm{~ms}, M=4, T=125.0 \mathrm{~ms}$, and number of OFF- and ON-cycles $=3$ and 2 . The first question we addressed was to look for an "optimal" stimulation amplitude. An optimal stimulation amplitude results in sufficiently strong effects, i.e., desynchronization of the target structure, while using a minimum stimulation amplitude to avoid potential side effects. Therefore, we increased the applied stimulation amplitude in a stepwise manner and investigated the desychronizing effect of the respective stimulation current amplitude $\kappa$. We performed this investigation for fixed electrode contact locations in the center of the STN volume. The value of the local order parameter $\overline{r_{1}}$ obtained after stimulation application is used as a quantitative measure for the desynchronization capabilities of a particular stimulation amplitude. The order parameter was averaged in time (interval $t \in[1140,1200] \mathrm{s}$, i.e., the last $60 \mathrm{~s}$ of simulated neuronal activity). The averaging started $420 \mathrm{~s}$ after cessation of stimulation. Figure 10 illustrates the dependence of the local order parameter $\bar{r}_{1}$ on the stimulation amplitude $\kappa$. The illustration shows that for low stimulation amplitudes a significant decrease in synchronized activity could not be achieved. For instance, with an amplitude of $\kappa=-1.0 \mathrm{~mA}$ the system remained in a strongly synchronized state after stimulation, and the low standard deviation for $\bar{r}_{1}$ indicated that the entire STN volume was homogeneously synchronized. This was because for these low amplitudes the stimulation signal was not strong enough, and neurons in further distance from the electrode contacts did not sense a sufficiently strong input current in order to achieve a reliable phase reset. Hence, the amount of neurons recruited by stimulation was not large enough for very low stimulation amplitudes.

With further increasing amplitude, the fraction of neurons sensing significant stimulation inputs linearly increased and the electrical CR stimulation unfolded its desynchronizing effect. Nevertheless, large standard deviations of $\bar{r}_{1}$ for stimulation
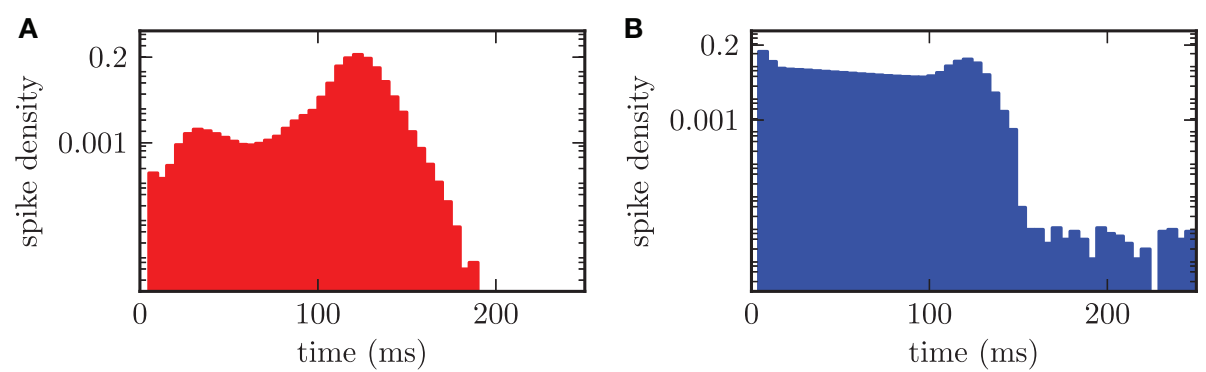

FIGURE 9 | ISI histogram (semi-logarithmic plot) for both neuronal populations in the synchronized state. (A) The STN neurons synchronize with a median period of $\tilde{T}_{\text {stn }}=122.0 \mathrm{~ms}$. (B) Two maxima in the ISI histogram of the GPe population due to bursting activity of the GPe neurons and the external forcing from the STN. 


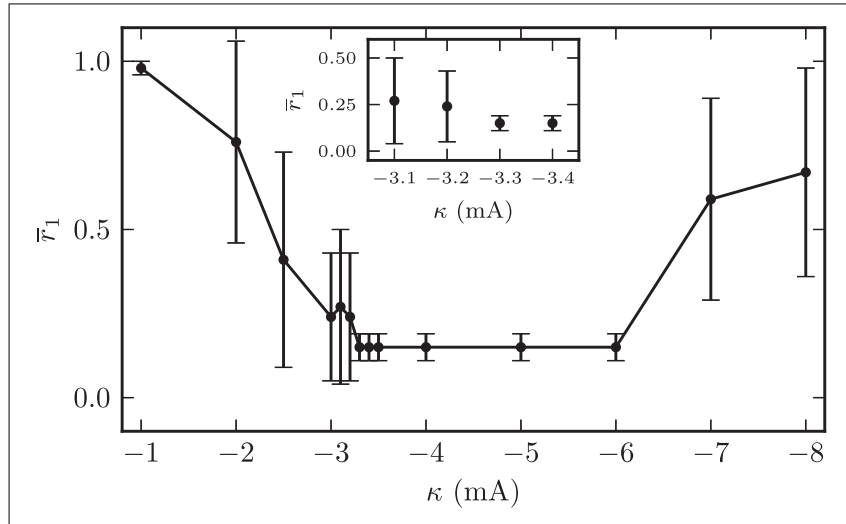

FIGURE 10 | Dependence of the local synchronization parameter $\bar{r}_{1}$ measured $420 \mathrm{~s}$ after cessation of stimulation on the used stimulation current amplitude. The plot demonstrates an amplitude range associated with a complete desynchronization of the STN volume.

amplitudes below $-3.2 \mathrm{~mA}$ indicated the existence of large discrepancies in the spatial distribution of the local order parameters. For illustration, the effects of CR stimulation with amplitude $\kappa=-2.0 \mathrm{~mA}$ on the model system are shown in Figure 11. The mean synaptic weight was successfully decreased during stimulation, but not sufficiently enough in order to shift the system into an overall strongly desynchronized state. After the stimulation the mean synaptic weight increased again and stabilized at $\bar{w}_{\mathrm{ss}}(t \rightarrow \infty) \approx 10.4 \cdot 10^{-3}$. Accordingly, the order parameters were reduced, but both values after stimulation $\left(\left\langle R_{1}\right\rangle_{\mathrm{stn}}=0.7\right.$, $\left.\left\langle R_{1}\right\rangle_{\text {gpe }}=0.63\right)$ indicated the persistence of reduced, but still highly correlated activity within the STN and GPe sub-networks. Figure 11C shows the spatial distribution of the local order parameter after CR stimulation and provides an explanation for these observations. Only the inner part of the STN volume was reliably desynchronized, while synaptic connections and thus synchronization among neurons at larger distances from the electrode could not be reduced due to an insufficient stimulation amplitude. However, these patterns of local (de-)synchronization after CR application were stable within our observation times. Above we showed that for spatially homogeneous initial conditions the system approaches one of the two dominating attractors with strongly synchronized activity (high mean coupling strength) or strongly desynchronized activity (low mean coupling strength) cf. Figure 4B. Our observations show that the coupling strength between neurons can be influenced locally by electrical CR stimulation and thus the system can be forced to approach one of the stable attractors shown in Figure 4B if the fraction of manipulated connections is large enough.

This figure also indicates that the synaptic connection strengths between STN neurons, which were governed by a STDP learning rule, converged either to values close to the maximum synaptic weight $\bar{w}_{\mathrm{ss}}^{\max }=20.0 \cdot 10^{-3}$ or to values close to the minimum of $\bar{w}_{\mathrm{ss}}^{\mathrm{min}}=0$. The minimal stimulation amplitude, which was capable to achieve a persistent desynchronization of the complete STN volume, was $\kappa_{\text {opt }}=-3.3 \mathrm{~mA}$. The stimulation outcome did not differ qualitatively for stimulation amplitudes above $\kappa_{\text {opt }}$. However, the effective desynchronization of the STN volume broke down for amplitudes above $-6.0 \mathrm{~mA}$. This effect could be explained by a reduced spatial specificity of the stimulation for larger amplitudes, i.e., the regions stimulated by the different electrode contacts showed high intersections among each other, which led to stimulation of the entire STN population instead of small sub-populations of STN neurons.

For intermediate amplitudes (i.e., $\kappa \in[-3.3 \mathrm{~mA},-6.0 \mathrm{~mA}]$ ) and during active $\mathrm{CR}$ stimulation the STN is divided into four synchronized sub-populations. The four synchronized subpopulations origin from the four stimulation sites, i.e., the periodic stimulation at each individual stimulation site causes a local synchronization of the surrounding neurons. Through the four stimulation sites a phase-shifted stimulation pattern (i.e., the typical CR pattern) is applied, which results in an overall weakening of synaptic weights and thus decreases the synchronized activity of the whole STN population already during stimulation.

For larger amplitudes almost all STN neurons are synchronized and form one population which is firing in phase. This synchronized firing does not decrease synaptic weights.

In Figure 12 we illustrated the effects of CR stimulation at appropriate intensity, by considering the results obtained with the minimal stimulation amplitude enabling complete desynchronization, $\kappa_{\mathrm{opt}}=-3.3 \mathrm{~mA}$. Before the stimulation was switched on the mean synaptic weight among STN neurons was stable and close to its maximum value. During the application of CR stimulation the mean weight $\bar{w}_{\text {ss }}$ monotonically decayed. After 10 min the continuous CR stimulation was switched off and without further stimulation the mean synaptic weight stabilized close to zero (Figure 9A). The order parameters for the STN and the GPe population decreased during stimulation, but saturated and fluctuated around a value of approximately $R_{1}=0.35$. This is due to the fact that during application of CR stimulation sub-populations of the STN are periodically synchronized before they undergo a desynchronization transient. Nevertheless, after switching stimulation off both neuronal populations fired in a strongly desynchronized manner and remained in this state.

\subsection{INFLUENCE OF ELECTRODE LOCATION}

In order to investigate the influence of the stimulation electrode position within the STN volume on the desynchronizing effect of CR stimulation, we varied the electrode position, i.e., the location of the array of stimulation contacts and analyzed the stimulation outcome in comparison to an ideal reference position. The topology of the array of stimulation contacts remained constant. The electrode position with the four electrode contacts centered in the STN volume served as reference position. Starting from this reference position, we consecutively moved the electrode along the three respective coordinate axes out of the center of the target in steps of $1.0 \mathrm{~mm}$. For all simulations we used the stimulation amplitude of $\kappa=-3.3 \mathrm{~mA}$ and the same stimulation parameters as before. The mean local order parameter $\bar{r}_{1}$ was computed over the last $60 \mathrm{~s}$ of simulated neuronal activity $t \in[1140,1200] \mathrm{s}$ and after cessation of stimulation. In Figure 13 we display the dependence of the local order parameter $\bar{r}_{1}$ after CR stimulation on the displacement $D$ along the three respective coordinate axes from the reference position. With the electrode located at the reference position the desynchronization of the STN volume was 

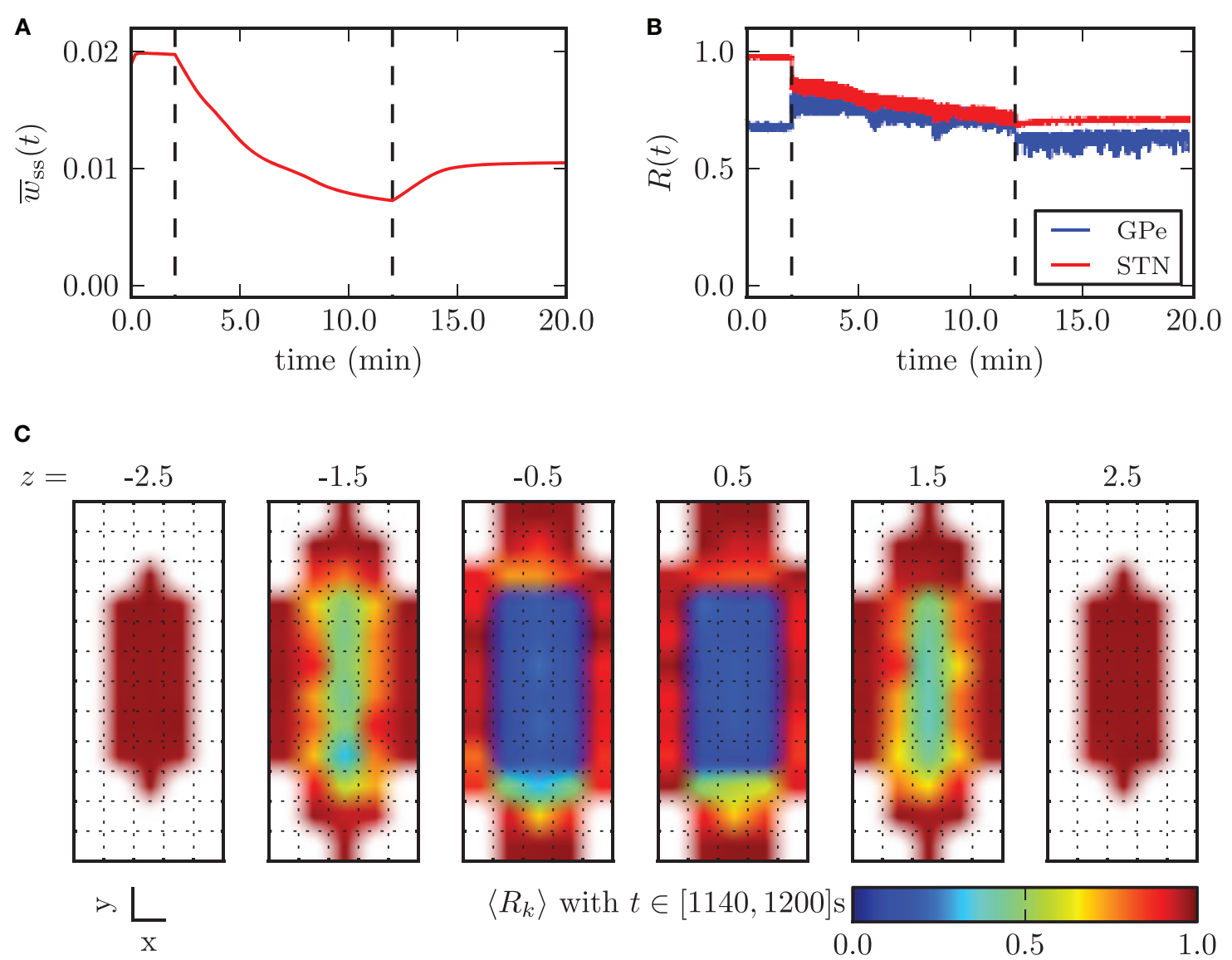

FIGURE 11 | Outcome of CR stimulation with insufficient amplitude $(\boldsymbol{\kappa}=\mathbf{- 2 . 0} \mathbf{m A})$. The dashed lines indicate when the stimulation was switched on/off. (A) The mean synaptic weight decreased during stimulation, but this effect did not persist after cessation of stimulation, after which the mean weight slightly recovered. (B) The order parameter within the STN population was reduced, while it stayed at the same level in the GPe, but with larger fluctuations. (C) shows that desynchronized neuronal activity was restored only within the inner core of the STN volume. most effective. With further distance from the reference position the local order parameter $\bar{r}_{1}$ increased monotonically for growing displacements of the electrode irrespective of the considered coordinate axis.

The dependence of $\bar{r}_{1}$ on the displacement along the $x$ - and $z$ axis was very similar. This was caused by the fact that the ellipsoid defining the boundary of the STN volume was almost symmetrical in $x$-and $z$-direction with values for the semi-principal axes of $a_{\text {stn }}=2.5 \mathrm{~mm}$ and $c_{\text {stn }}=3.0 \mathrm{~mm}$. The slope of the increase in $\bar{r}_{1}$ was significantly lower for the displacement along the $y$ axis, because the volume of neurons stimulated with a sufficiently high amplitude decreased with a lower rate than for displacement of the electrode along the two other coordinate axes. Therefore, we can conclude that the effect of electrode displacement on the stimulation outcome had a more significant impact for deviations along the $x$ - and $z$-axes than along the $y$-axis.

However, as mentioned before, the increase in stimulation amplitude is limited by several factors. First, an effective desynchronization could not be achieved for amplitudes which exceeded a certain (model dependent) threshold, as showed above. Second, larger stimulation amplitudes might induce unwanted side effects via unintentional stimulation of other brain areas or fiber tracts (Smith et al., 2012). Third, high stimulation current amplitudes might induce permanent damage to the neuronal tissue surrounding the electrode (Grill and McIntyre, 2001; Grill, 2005; Cogan, 2008).

\section{DISCUSSION}

In this study we developed a large-scale model of two basal ganglia structures STN and GPe, which are hypothesized to generate rhythmic synchronized oscillations. This synchronized activity is associated with symptoms arising in PD (Lenz et al., 1994; Plenz and Kital, 1999). The described model is the continuation of our top-down approach (Tass, 1999, 2003c; Popovych et al., 2005; Tass and Majtanik, 2006; Hauptmann and Tass, 2007, 2009, 2010; Tass and Hauptmann, 2009). By gradually increasing the complexity of our model, we were able to link our findings to previous results obtained from models with a reduced level of detail. Therefore, we increased the model size, parallelized the simulations and data analysis. Thus we were able to achieve a simulation performance close to real-time. We investigated and characterized the two predominant stable synchronized 

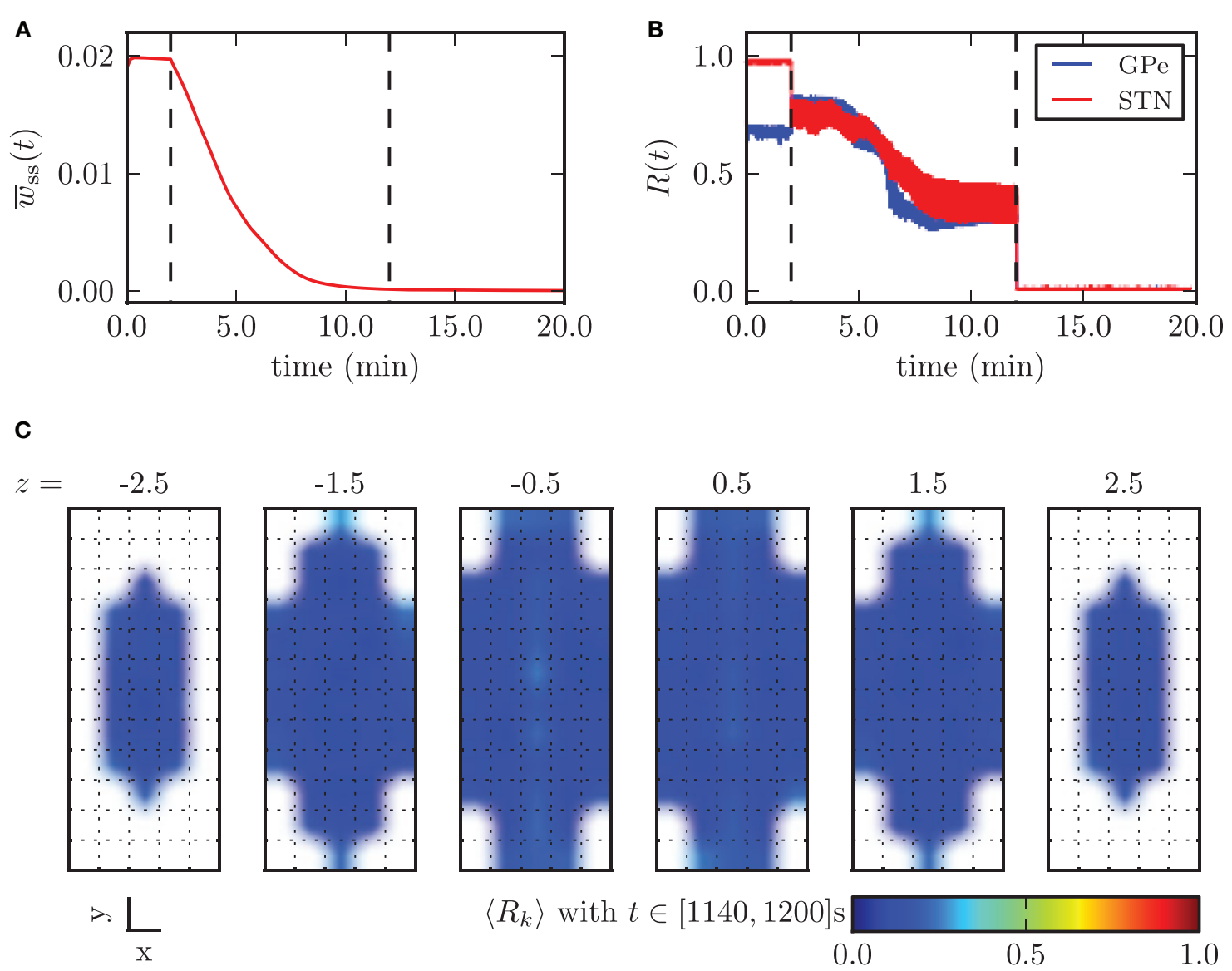

FIGURE 12 | Outcome of CR stimulation with appropriate amplitude ( $\boldsymbol{\kappa}=\mathbf{- 3 . 3} \mathbf{~ m A}$ ). (A) Mean synaptic weight among STN neurons and (B) time course of the order parameter. The points in time when the stimulation was switched on and off are marked by the vertical dashed lines. (C) shows the spatial distribution of synchronization and illustrates the homogeneous desynchronization in the whole STN volume. and desynchronized states, which modeled the pathological and healthy state of the basal ganglia system, respectively. We showed that the transition between both predominant states was dependent on the mean coupling strength among STN neurons. This observation is in good accordance with previous modeling studies (Hauptmann and Tass, 2007, 2009; Tass and Hauptmann, 2007, 2009). In addition, with our large-scale approach we were able to reveal clinically relevant spatio-temporal effects: spatially incomplete desynchronization caused by either insufficient stimulation or by electrode displacement.

Furthermore, we investigated the effects of CR stimulation on the network activity and, in particular, on the synaptic structure of the STN sub-network. In this context we were especially interested in how the neuronal network can be shifted from the strongly synchronized to the desynchronized mode of network activity. To this end, we introduced a synaptic learning rule and a measure enabling to assess the distribution of synchronized neuronal activity within the volume of the STN. In our investigations of the desynchronizing CR stimulation technique, we observed a certain amplitude range for which desynchronization of the target structure was most effective. This finding might be useful for further experimental studies. This optimal parameter range has been reported previously for simpler models of coupled phase oscillators, FitzHugh-Nagumo spiking neurons, FitzHugh-Rinzel bursting neurons, and Hodgkin-Huxley neurons with and without STDP (Guo and Rubin, 2011; Lysyansky et al., 2011; Popovych and Tass, 2012). Additionally, in an experimental study in parkinsonian non-human primates it was shown that CR DBS at low intensity has long-lasting after effects on basal ganglia motor function (Tass et al., 2012b) and a clinical proof-of-concept trial revealed that CR DBS has lasting aftereffects in patients with Parkinsons disease as assessed by UPDRS III scores and local field potential theta and beta activity (Adamchic et al., 2014a).

In this study we expanded these observations in a more complex and microanatomically more realistic model. We incorporated more realistic coupling topologies and took the spatial arrangement of the electrode and stimulated neurons into account. However, the observed amplitude parameters highly depend on how the electric field surrounding the electrode is modeled (Richardson et al., 2003; Butson et al., 2006; Chaturvedi et al., 2010; Schmidt and van Riemen, 2012). Therefore, a generalized quantitative prediction of optimal stimulation parameters is difficult to obtain from a theoretical simulation study. Additionally, the spatial structure enabled us to vary the electrode 

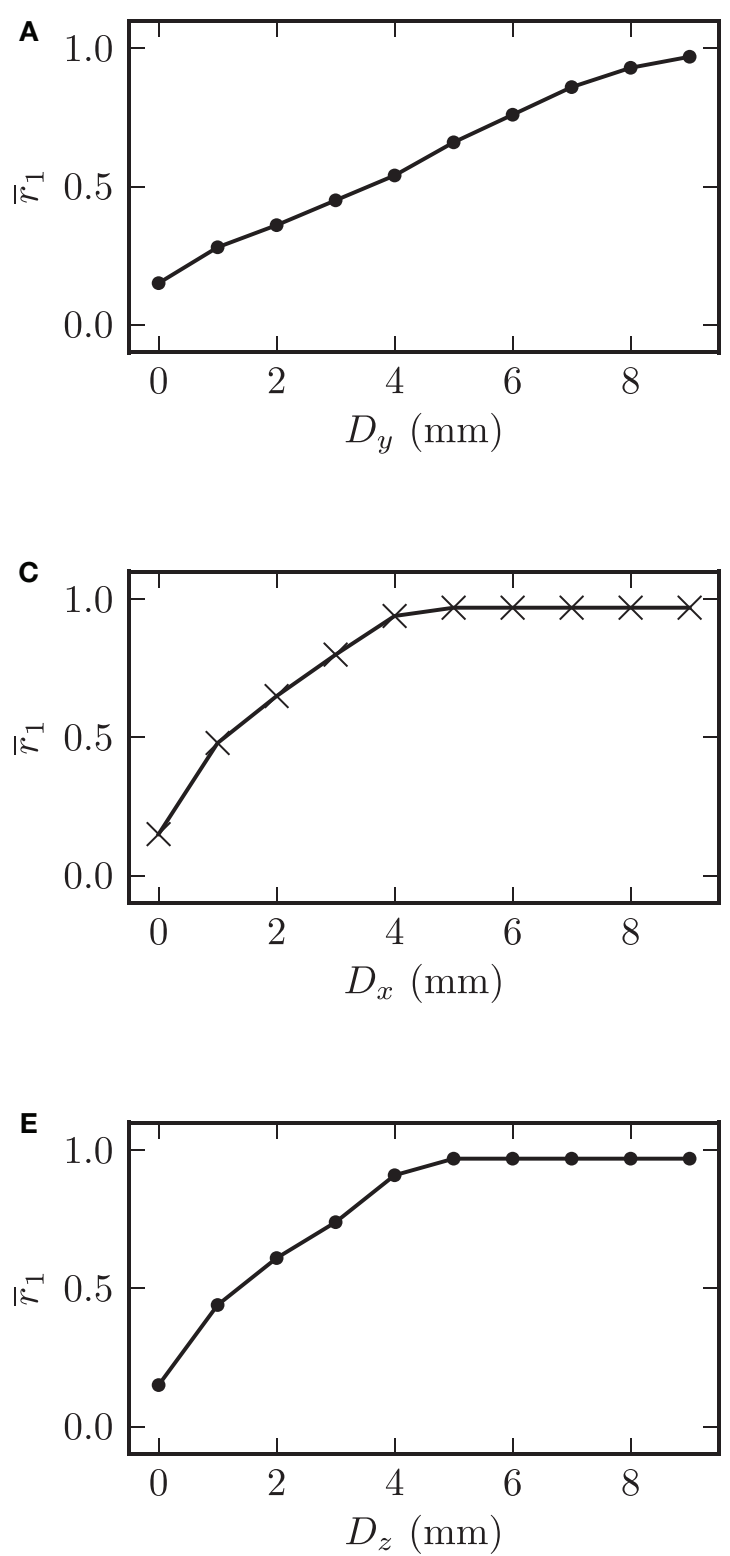

FIGURE 13 | On the left hand side the displacement of the electrode along the respective coordinate axis from the reference position in the center of the STN volume is plotted against the mean local order parameter $\overline{\boldsymbol{r}}_{\mathbf{1}}$ after stimulation. The $y$-axis points into the direction of electrode implantation. On the right hand side the spatial distribution of the local order parameter is shown for specific values of $D$. (A) electrode

\section{B}
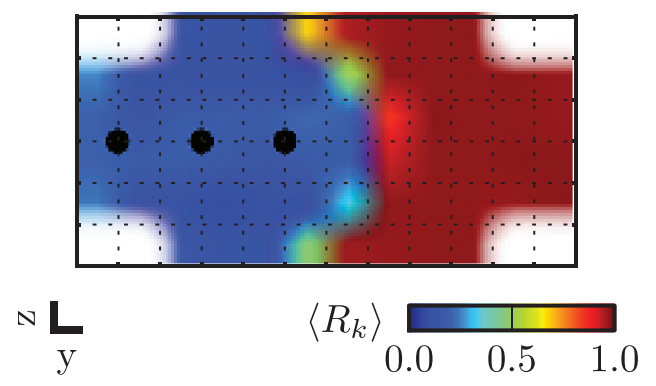

D

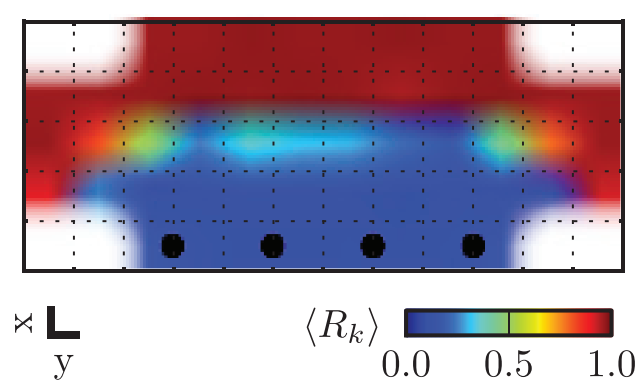

$\mathbf{F}$
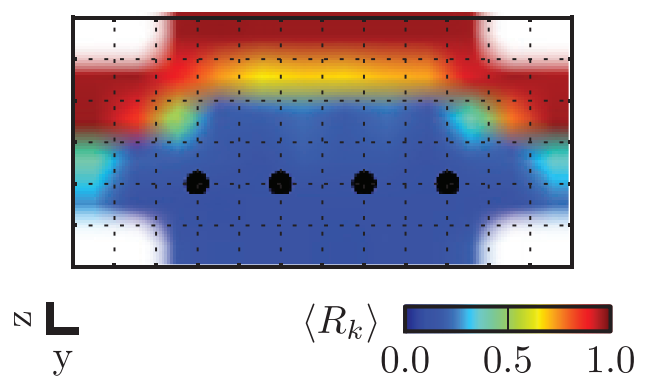

displacement along $y$-axis vs. local order parameter (B) example for $D_{y}=4.0 \mathrm{~mm}$ (C) electrode displacement along $x$-axis vs. local order parameter (D) example for $D_{x}=2.0 \mathrm{~mm}$ (E) electrode displacement along $z$-axis vs. local order parameter (F) example for $D_{z}=1.0 \mathrm{~mm}$. Plots (B) and (F) show a cross-section of the (y,z)-plane with $x=0 \mathrm{~mm}$, and (D) displays a cross-section through the $(x, y)$-plane with $z=-0.5, \mathrm{~mm}$. location within the stimulation target and thus we observed a strong relation between the electrode position and the stimulation outcome. This observation substantiates previous theoretical and experimental studies, which investigated the impact of the electrode position on the stimulation outcome for HF DBS (Voges et al., 2002; Paek et al., 2011; Nakano et al., 2012; Reese et al., 2012; Guo et al., 2013).

Because of the high computational costs of our complex model, we limited our observations of CR DBS effects to timescales of several 10 of minutes. Therefore, we used a STDP learning rule which showed adequate effects on such timescales. Our model could adequately reproduce the experimentally observed long-term desynchronization effects of $\mathrm{CR}$ stimulation, which last on timescales from minutes to hours and days, demonstrated in slice experiments (Tass et al., 2009), a pre-clinical proof-of-concept study (Tass et al., 2012b) and a proof-of-concept trial (Adamchic et al., 2014a). Certainly, the learning rule has a significant impact on the simulation results. 
Therefore, more experimental data specifying the actual plasticity mechanisms in the basal ganglia and especially within the STN are required. In order to be able to simulate the long-term effects (timescales of days or weeks) of CR DBS, a modification of the STDP rule has to be considered. However, these long-term simulations necessitate a further improvement of the simulation code in terms of scalability and performance or a reduction of the model complexity. At this point less complex models might be more suitable for the investigation of long-term DBS effects, but with steadily increasing performance and computing capacity these long-term simulations will be possible in the medium-term.

We considered many physiologically relevant parameters that have been neglected in former simplified models of the STNGPe network (e.g., spatial extent, realistic neuronal connectivity, and dimensionful neuron model) (Hauptmann et al., 2005; Feng et al., 2007; Tass and Hauptmann, 2009; Guo and Rubin, 2011; Lysyansky et al., 2011). This complex modeling approach had the advantage that characteristics observed in the model activity might be more transferable to an experimental verification. However, there are still some limitations and simplifications in our model. We used a simplified approximation for the distance dependence of the electric field (and thus the stimulation strength) surrounding the electrode. Besides the assumption that the electric field strength behaves similar to the radial component of the field for a finite line charge, this approximation was based on a homogeneous and isotropic tissue conductance. In a future refinement of the presented model the implementation of more realistic representations of the electric field (e.g., based on finite element methods McIntyre et al., 2004b) is intended (Butson and McIntyre, 2005, 2006; Butson et al., 2006; Chaturvedi et al., 2010; Schmidt and van Riemen, 2012). However, if a "realistic" representation is desired it is also necessary to consider the movements of charge carriers in the surrounding tissue (e.g., extracellular fluid). Obviously, substantiation of such a modeling approach requires more experimental data. Also, in this study we trade-off model complexity, to reduce computational costs. Accordingly, we introduced STDP only within the STN, since this is the primary stimulation target for CR DBS.

By the same token, future studies might incorporate more complex STDP rules including soft bounds (van Rossum et al., 2000). The effects of hard vs. soft bounds in a similar system were studied in detail in Popovych et al. (2013). That study provides a systematical investigation of the effects of hard and soft bounds on spontaneous dynamical states (in particular, multistability) as well as stimulation-driven dynamical states (stochastic resonance induced by uncorrelated noise) of a network. The stochastic resonance was robust with respect to these modifications. Furthermore, multistability, an essential ingredient for $\mathrm{CR}$ to induce long-lasting effects, as well as robust anti-kindling was observed for hard bounds (Tass and Majtanik, 2006) as well as for soft bounds (Maistrenko et al., 2007) in phase oscillator networks. Correspondingly, to demonstrate that the CR-induced anti-kindling does not depend on the particular choice of bounds in the model used in this paper, among other future developments we are planning to take into account soft bounds in a forthcoming study, too. Moreover, further studies might be used to investigate more complex phenomena in the field of STDP, for example the observation that in the presence of propagation delays in recurrent networks exhibiting population bursts, STPD rules might exert a strong decoupling force which can further the desynchronization of the network activity (Lubenov and Siapas, 2008). Also, STDP can induce oscillatory behaviors depending also on the model parameters and in particular on the shape of the considered pulse (Mikkelsen et al., 2014). Further studies might also incorporate neighboring structures which are omitted in the current approach. For example, GPe fibers and axonal pathways, which might be relevant for a detailed analysis of the effects of variations of the electrode position (Rubin et al., 2012). Additional measures, focussing on the effect of information transfer on antidromic structures might further increase the understanding of the therapeutic effect of different sites and types of stimulation (Rubin and Terman, 2004; Rubin et al., 2012). Note, optimal electrode placement for standard HF DBS might differ quite substantially from that of CR DBS. Computational results show that CR is effective no matter whether neurons are stimulated at somata or via inhibitory or excitatory synapses (Popovych and Tass, 2012). Accordingly, in our paper we concentrated on the central electrode placement, since from a clinical standpoint it might be feasible already with available depth electrodes.

Our investigations of different electrode positions showed that the placement of the stimulation electrode within the target structure is crucial for an effective desynchronization. Furthermore, the required stimulation current amplitude was dependent on the electrode location. In the context of a clinically more effective electrode placement it is still under discussion, whether the optimal stimulation site for HF DBS lies within the interior of the STN or in fiber tracts located close to the STN (Voges et al., 2002; Paek et al., 2011; Nakano et al., 2012; Reese et al., 2012; Guo et al., 2013). In order to tackle this issue utilizing a modeling approach, a future version of our model should include spatially extended multi-compartment neurons instead of pointlike model neurons. Hence, the mentioned fiber tracts should be represented by multi-compartment cable models.

Another feature of DBS relevant to possible further improvement is the shape of the stimulation pulses used. In various studies the efficacy of particular pulse shapes for neuronal stimulation was investigated (Kuncel and Grill, 2004; Kuncel et al., 2006; Butson and McIntyre, 2007; Foutz and McIntyre, 2010; Wongsarnpigoon and Grill, 2010; Hofmann et al., 2011). Apart from the stimulation pulse shapes, the other CR parameters (e.g., stimulation frequency, intra-burst frequency etc.) deserve further detailed investigations. Accordingly, our work provides a tool to probe related stimulation effects, which might be observed experimentally in future pre-clinical and clinical studies. Our model might contribute to a clinically useful computational tool for the optimization of parameters for CR DBS. In particular, the spatially extented topology of our model might be important for the development of new stimulation electrode geometries (Buhlmann et al., 2011; Martens et al., 2011).

\section{ACKNOWLEDGMENTS}

We acknowledge valuable comments by Klaus Lehnertz. We thank Hans E. Plesser, Markus Diesmann, and the entire NEST 
community for providing an easy to use and extendable software and their advice. We thank Judith Coenen, Harald Treuer, Mohammad Maarouf, and Volker Sturm for providing us with the MRI segmentation data. This study was supported by the Helmholtz Alliance on Systems Biology and the Bonn Cologne Graduate School of Physics and Astronomy (BCGS), which is funded by the Deutsche Forschungsgemeinschaft (DFG).

\section{REFERENCES}

Adamchic, I., Hauptmann, C., Barnikol, U., Pawelczyk, N., Popovych, O., Barnikol, T., et al. (2014a). Coordinated reset neuromodulation for Parkinson's disease: proof-of-concept study. Mov. Disord. 29, 1679-1684. doi: 10.1002/mds. 25923

Adamchic, I., Toth, T., Hauptmann, C., and Tass, P. A. (2014b). Reversing pathologically increased EEG power by acoustic CR neuromodulation. Hum. Brain Mapp. 35, 2099-2118. doi: 10.1002/hbm.22314

Afsharpour, S. (1985). Light microscopic analysis of golgi-impregnated rat subthalamic neurons. J. Comp. Neurol. 236, 1-13. doi: 10.1002/cne.902360102

Anderson, R., Frye, M., Abulseoud, O., Lee, K., McGillivray, J., Berk, M., et al. (2012). Deep brain stimulation for treatment-resistant depression: efficacy, safety and mechanisms of action. Neurosci. Biobehav. Rev. 36, 1920-1933. doi: 10.1016/j.neubiorev.2012.06.001

Baufreton, J., and Bevan, M. D. (2008). D2-like dopamine receptor-mediated modulation of activity-dependent plasticity at GABAergic synapses in the subthalamic nucleus. J. Physiol. 586, 2121-2142. doi: 10.1113/jphysiol.2008. 151118

Baufreton, J., Kirkham, E., Atherton, J. F., Menard, A., Magill, P. J., Bolam, J. P., et al. (2009). Sparse but selective and potent synaptic transmission from the globus pallidus to the subthalamic nucleus. J. Neurophysiol. 102, 532-545. doi: 10.1152/jn.00305.2009

Benabid, A. L., Pollak, P., Gervason, C., Hoffmann, D., Gao, D. M., Hommel, M., et al. (1991). Long-term suppression of tremor by chronic stimulation of the ventral intermediate thalamic nucleus. Lancet 337, 403-406. doi: 10.1016/01406736(91)91175-T

Bevan, M. D., Magill, P. J., Terman, D., Bolam, J. P., and Wilson, C. J. (2002). Move to the rhythm: oscillations in the subthalamic nucleus-external globus pallidus network. Trends Neurosci. 25, 525-531. doi: 10.1016/S0166-2236(02) 02235-X

Bi, G.-Q., and Poo, M.-M. (1998). Synaptic modifications in cultured hippocampal neurons: Dependence on spike timing, synaptic strength, and postsynaptic cell type. J. Neurosci. 18, 10464-10472.

Bi, G.-Q., and Poo, M.-M. (2001). Synaptic modification by correlated activity: Hebb's postulate revisited. Annu. Rev. Neurosci. 24, 139-166. doi: 10.1146/annurev.neuro.24.1.139

Brown, P., Oliviero, A., Mazzone, P., Insola, A., Tonali, P., and Lazzaro, V. D. (2001). Dopamine dependency of oscillations between subthalamic nucleus and pallidum in Parkinson's disease. J. Neurosci. 21, 1033-1038.

Buhlmann, J., Hofmann, L., Tass, P. A., and Hauptmann, C. (2011). Modeling of a segmented electrode for desynchronizing deep brain stimulation. Front. Neuroeng. 4:15. doi: 10.3389/fneng.2011.00015

Butson, C. R., Maks, C. B., and McIntyre, C. C. (2006). Sources and effects of electrode impedance during deep brain stimulation. Clin. Neurophysiol. 117, 447-454. doi: 10.1016/j.clinph.2005.10.007

Butson, C. R., and McIntyre, C. C. (2005). Tissue and electrode capacitance reduce neural activation volumes during deep brain stimulation. Clin. Neurophysiol. 16, 2490-2500. doi: 10.1016/j.clinph.2005.06.023

Butson, C. R., and McIntyre, C. C. (2006). Role of electrode design on the volume of tissue activated during deep brain stimulation. J. Neural Eng. 3, 1-8. doi: 10.1088/1741-2560/3/1/001

Butson, C. R., and McIntyre, C. C. (2007). Differences among implanted pulse generator waveforms cause variations in the neural response to deep brain stimulation. Clin. Neurophysiol. 118, 1889-1894. doi: 10.1016/j.clinph.2007. 05.061

Cannon, E., Silburn, P., Coyne, T., O’Maley, K., Crawford, J. D., and Sachdev, P. S. (2012). Deep brain stimulation of anteromedial globus pallidus interna for severe Tourette's syndrome. Am. J. Psychiatry 169, 860-866. doi: 10.1176/appi.ajp.2012.11101583
Chaturvedi, A., Butson, C. R., Lempka, S. F., Cooper, S. E., and McIntyre, C. C. (2010). Patient-specific models of deep brain stimulation: influence of field model complexity on neural activation predictions. Brain Stimul. 3, 65-77. doi: 10.1016/j.brs.2010.01.003

Coffey, R. J. (2009). Deep brain stimulation devices: a brief technical history and review. Arti. Organs 33, 208-220. doi: 10.1111/j.1525-1594.2008. 00620.x

Cogan, S. F. (2008). Neural stimulation and recording electrodes. Annu. Rev. Biomed. Eng. 10, 275-309. doi: 10.1146/annurev.bioeng.10.061807. 160518

Daido, H. (1992). Order function and macroscopic mutual entrainment in uniformly coupled limit-cycle oscillators. Prog. Theor. Phys. Suppl. 88, 1213-1218. doi: $10.1143 / \mathrm{ptp} / 88.6 .1213$

Dayan, P., and Abbott, L. (2000). Theoretical Neuroscience. Cambridge, MA: The MIT Press.

Deuschl, G., Raethjen, J., Baron, R., Lindemann, M., Wilms, H., and Krack, P. (2000). The pathophysiology of Parkinsonian tremor: A review. J. Neurol. 247, 33-48. doi: 10.1007/PL00007781

Deuschl, G., Schade-Brittinger, C., Krack, P., Volkmann, J., Schäfer, H., Bötzel, K., et al. (2006). A randomized trial of deep-brain stimulation for Parkinson's disease. N. Engl. J. Med. 355, 896-908. doi: 10.1056/NEJMoa060281

Eppler, J. M., Helias, M., Muller, E., Diesmann, M., and Gewaltig, M.-O. (2008). PyNEST: a convenient interface to the NEST simulator. Front. Neuroinform. 2:12. doi: 10.3389/neuro.11.012.2008

Fahn, S. (2008). The history of dopamine and levodopa in the treatment of Parkinson's disease. Move. Disord. 23, 497-508. doi: 10.1002/mds.22028

Fehlberg, E. (1970). Klassische runge-kutta-formeln vierter und niedrigerer ordnung mit schrittweiten-kontrolle und ihre anwendung auf Wärmeleitungsprobleme. Computing 6, 61-71. doi: 10.1007/BF022 41732

Feldman, D. (2000). Timing based LTP and LTD at vertical inputs to layer ii/iii pyramidal cells in rat barrel cortex. Neuron 27, 45-56. doi: 10.1016/S08966273(00)00008-8

Feng, X.-J., Shea-Brown, E., Greenwald, B., Kosut, R., and Rabitz, H. (2007). Optimal deep brain stimulation of the subthalamic nucleus-a computational study. J. Comput. Neurosci. 23, 265-282. doi: 10.1007/s10827-007-0031-0

Foutz, T. J., and McIntyre, C. C. (2010). Evaluation of novel stimulus waveforms for deep brain stimulation. J. Neural Eng. 7:066008. doi: 10.1088/17412560/7/6/066008

Fujimoto, K., and Kita, H. (1993). Response characteristics of subthalamic neurons to the stimulation of the sensorimotor cortex in the rat. Brain Res. 23, 185-192. doi: 10.1016/0006-8993(93)90872-K

Galassi, M., Davies, J., Theiler, J., Gough, B., Jungman, G., Alken, P., et al. (2009). GNU Scientific Library Reference Manual - Third Edition. Godalming: Network Theory Ltd.

Gerstner, W., Kempter, R., van Hemmen, J. L., and Wagner, H. (1996). A neuronal learning rule for sub-millisecond temporal coding. Nature 383, 76-78. doi: $10.1038 / 383076 \mathrm{a} 0$

Gerstner, W., and Kistler, W. M. (2002). Spiking Neuron Models. Cambridge: Cambridge University Press. doi: 10.1017/CBO9780511815706

Gewaltig, M.-O., and Diesmann, M. (2007). NEST (NEural Simulation Tool). Scholarpedia 2:1430. doi: 10.4249/scholarpedia.1430

Gillies, A. J., and Willshaw, D. J. (1998). A massively connected subthalamic nucleus leads to the generation of widespread pulses. Proc. R. Soc. Lond. B 265, 2101-2109. doi: 10.1098/rspb.1998.0546

Gillies, A. J., and Willshaw, D. J. (2004). Models of the subthalamic nucleus: the importance of intranuclear connectivity. Med. Eng. Phys. 26, 723-732. doi: 10.1016/j.medengphy.2004.06.003

Gillies, A. J., Willshaw, D. J., and Li, Z. (2002). Subthalamic-pallidal interactions are critical in determining normal and abnormal functioning of the basal ganglia. Proc. R. Soc. Lond. B 269, 545-551. doi: 10.1098/rspb.2001.1817

Gradinaru, V., Mogri, M., Thompson, K., Henderson, J., and Deisseroth, K. (2009). Optical deconstruction of Parkinsonian neural circuitry. Science 324, 354-359. doi: 10.1126/science.1167093

Grill, W. M. (2005). Safety considerations for deep brain stimulation: review and analysis. Exp. Rev. Med. Dev. 2, 409-420. doi: 10.1586/17434440.2.4.409

Grill, W. M., and McIntyre, C. C. (2001). Extracellular excitation of central neurons: implications for the mechanisms of deep brain stimulation. Thalamus Relat. Syst. 1, 269-277. doi: 10.1017/S1472928801000255 
Guo, S., Zhuang, P., Hallett, M., Zheng, Z., Zhang, Y., Li, J., et al. (2013). Subthalamic deep brain stimulation for Parkinson's disease: correlation between locations of oscillatory activity and optimal site of stimulation. Parkinsonism Rel. Disord. 19, 109-114. doi: 10.1016/j.parkreldis.2012.08.005

Guo, Y., and Rubin, J. E. (2011). Multi-site stimulation of subthalamic nucleus diminishes thalamocortical relay errors in a biophysical network model. Neural Netw. 24, 602-616. doi: 10.1016/j.neunet.2011.03.010

Hauptmann, C., Popovych, O., and Tass, P. A. (2005). Effectively desynchronizing deep brain stimulation based on a coordinated delayed feedback stimulation via several sites: a computational study. Biol. Cybernet. 93, 463-470. doi: 10.1007/s00422-005-0020-1

Hauptmann, C., and Tass, P. A. (2007). Therapeutic rewiring by means of desynchronizing brain stimulation. Biosystems 89, 173-181. doi: 10.1016/j.biosystems.2006.04.015

Hauptmann, C., and Tass, P. A. (2009). Cumulative and after-effects of short and weak coordinated reset stimulation: a modeling study. J. Neural Eng. 6, 1-13. doi: 10.1088/1741-2560/6/1/016004

Hauptmann, C., and Tass, P. A. (2010). Restoration of segregated neuronal activity by desynchronizing stimulation in the presence of sensory input. J. Neural Eng. 7:056008. doi: 10.1088/1741-2560/7/5/056008

Hellwig, B. (2000). A quantitative analysis of the local connectivity between pyramidal neurons in layers $2 / 3$ of the rat visual cortex. Biol. Cybernet. $82,111-121$. doi: 10.1007/PL00007964

Hofmann, L., Ebert, M., Tass, P. A., and Hauptmann, C. (2011). Modified pulse shapes for effective neural stimulation. Front. Neuroeng. 4:9. doi: 10.3389/fneng.2011.00009

Holgado, A. J. N., Terry, J. R., and Bogacz, R. (2010). Conditions for the generation of beta oscillations in the subthalamic nucleus-globus pallidus network. $J$. Neurosci. 30, 12340-12352. doi: 10.1523/JNEUROSCI.0817-10.2010

Humphries, M. D., and Gurney, K. N. (2012). Network effects of subthalamic deep brain stimulation drive a unique mixture of responses in basal ganglia output. Eur. J. Neurosci. 36, 2240-2251. doi: 10.1111/j.1460-9568.2012.08085.x

Kita, H., Chang, H., and Kitai, S. T. (1983). The morphology of intracellularly labeled rat subthalamic neurons: a light microscopic analysis. J. Comp. Neurol. 215, 245-257. doi: 10.1002/cne.902150302

Kita, H., Tachibana, Y., Nambu, A., and Chiken, S. (2005). Balance of monosynaptic excitatory and disynaptic inhibitory responses of the globus pallidus induced after stimulation of the subthalamic nucleus in the monkey. J. Neurosci. 25, 8611-8619. doi: 10.1523/JNEUROSCI.1719-05.2005

Kühn, A. A., Kempf, F., Brücke, C., Doyle, L. G., Martinez-Torres, I., Pogosyan, A., et al. (2008). High-frequency stimulation of the subthalamic nucleus suppresses oscillatory $\beta$-activity in patients with Parkinson's disease in parallel with improvement in motor performance. J. Neurosci. 28, 6165-6173. doi: 10.1523/JNEUROSCI.0282-08.2008

Kuncel, A. M., Cooper, S. E., Wolgamuth, B. R., Clyde, M. A., Snyder, S. A., Montgomery, E. B. Jr., et al. (2006). Clinical response to varying the stimulus parameters in deep brain stimulation for essential tremor. Move. Disord. 21, 1920-1928. doi: 10.1002/mds.21087

Kuncel, A. M., and Grill, W. M. (2004). Selection of stimulus parameters for deep brain stimulation. Clin. Neurophysiol. 115, 2431-2441. doi: 10.1016/j.clinph.2004.05.031

Kuramoto, Y. (1984). Chemical Oscillations, Waves, and Turbulence. Berlin: Springer. doi: 10.1007/978-3-642-69689-3

Lehnertz, K., Bialonski, S., Horstmann, M.-T., Krug, D., Rothkegel, A., Staniek, M., et al. (2009). Synchronization phenomena in human epileptic brain networks. J. Neurosci. Methods 183, 42-48. doi: 10.1016/j.jneumeth.2009.05.015

Lenz, F., Kwan, H., Martin, R., Tasker, R., Dostrovsky, J., and Lenz, Y. (1994). Single unit analysis of the human ventral thalamic nuclear group - Tremorrelated activity in functionally identified cells. Brain 117, 531-543. doi: 10.1093/brain/117.3.531

Levesque, J.-C., and Parent, A. (2005). GABAergic interneurons in human subthalamic nucleus. Move. Disord. 20, 574-584. doi: 10.1002/mds.20374

Limousin, P., Speelman, J. D., Gielen, F., and Janssens, M. (1999). Multicentre european study of thalamic stimulation in Parkinsonian and essential tremor. J. Neurol. Neurosurg. Psychiatry 66, 289-296. doi: 10.1136/jnnp. 66.3.289

Lubenov, E. V., and Siapas, A. G. (2008). Decoupling through synchrony in neuronal circuits with propagation delays. Neuron 58, 118-131. doi: 10.1016/j.neuron.2008.01.036
Lysyansky, B., Popovych, O. V., and Tass, P. A. (2011). Desynchronizing antiresonance effect of m:n ON-OFF coordinated reset stimulation. J. Neural Eng. 8:036019. doi: 10.1088/1741-2560/8/3/036019

Maistrenko, Y. L., Lysyansky, B., Hauptmann, C., Burylko, O., and Tass, P. A. (2007). Multistability in the Kuramoto model with synaptic plasticity. Phys. Rev. E 75:066207. doi: 10.1103/PhysRevE.75.066207

Mallet, N., Pogosyan, A., Márton, L. F., Bolam, J. P., Brown, P., and Magill, P. J. (2008). Parkinsonian beta oscillations in the external globus pallidus and their relationship with subthalamic nucleus activity. J. Neurosci. 28, 14245-14258. doi: 10.1523/JNEUROSCI.4199-08.2008

Markram, H., Lübke, J., Frotscher, M., and Sakmann, B. (1997). Regulation of synaptic efficacy by coincidence of postsynaptic APs and EPSPs. Science 275, 213-215. doi: 10.1126/science.275.5297.213

Martens, H. C. F., Toader, E., Decré, M. M. J., Anderson, D. J., Vetter, R., Kipke, D. R., et al. (2011). Spatial steering of deep brain stimulation volumes using a novel lead design. Clin. Neurophysiol. 122, 558-566. doi: 10.1016/j.clinph.2010.07.026

McIntyre, C. C., Grill, W. M., Sherman, D. L., and Thakor, N. V. (2004a). Cellular effects of deep brain stimulation: model-based analysis of activation and inhibition. J. Neurophysiol. 91, 1457-1469. doi: 10.1152/jn.00989.2003

McIntyre, C. C., Mori, S., Sherman, D. L., Thakor, N. V., and Vitek, J. L. (2004b). Electric field and stimulating influence generated by deep brain stimulation of the subthalamic nucleus. Clin. Neurophysiol. 115, 589-595. doi: 10.1016/j.clinph.2003.10.033

McIntyre, C. C., Savasta, M., Kerkerian-Le Goff, L., and Vitek, J. L. (2004c). Uncovering the mechanism(s) of action of deep brain stimulation: activation, inhibition, or both. Clin. Neurophysiol. 115, 1239-1248. doi: 10.1016/j.clinph.2003.12.024

Mikkelsen, K., Imparato, A., and Torcini, A. (2014). Sisyphus effect in pulsecoupled excitatory neural networks with spike-timing-dependent plasticity. Phys. Rev. E 89:062701. doi: 10.1103/PhysRevE.89.062701

Miocinovic, S., Parent, M., Butson, C. R., Hahn, P. J., Russo, G. S., Vitek, J. L., et al. (2006). Computational analysis of subthalamic nucleus and lenticular fasciculus activation during therapeutic deep brain stimulation. J. Neurophysiol. 96, 1569-1580. doi: 10.1152/jn.00305.2006

Montgomery, E. B. Jr., and Gale, J. T. (2008). Mechanisms of action of deep brain stimulation (DBS). Neurosci. Biobehav. Rev. 32, 388-407. doi: 10.1016/j.neubiorev.2007.06.003

Moro, E. E., Esselink, R. J., Xie, J., Hommel, M., Benabid, A. L., and Pollak, P. (2002). The impact on Parkinson's disease of electrical parameter settings in STN stimulation. Neurology 59, 706-713. doi: 10.1212/WNL.59.5.706

Morrison, A., Mehring, C., Geisel, T., Aertsen, A., and Diesmann, M. (2005). Advancing the boundaries of high-connectivity network simulation with distributed computing. Neural Comput. 17, 1776-1801. doi: 10.1162/0899766054026648

Nakano, N., Taneda, M., Watanabe, A., and Kato, A. (2012). Computed threedimensional atlas of subthalamic nucleus and its adjacent structures for deep brain stimulation in Parkinson's disease. ISRN Neurol. 2012, 1-13. doi: $10.5402 / 2012 / 592678$

Nini, A., Feingold, A., Slovin, H., and Bergman, H. (1995). Neurons in the globus pallidus do not show correlated activity in the normal monkey, but phase-locked oscillations appear in the MPTP model of Parkinsonism. J. Neurophysiol. 74, 1800-1805.

Nishibayashi, H., Ogura, M., Kakishita, K., Tanaka, S., Tachibana, Y., Nambu, A., et al. (2011). Cortically evoked responses of human pallidal neurons recorded during stereotactic neurosurgery. Move. Disord. 26, 469-476. doi: $10.1002 / \mathrm{mds} .23502$

Paek, S. H., Lee, J.-Y., Kim, H.-J., Kang, D., Lim, Y. H., Kim, M. R., et al. (2011). Electrode position and the clinical outcome after bilateral subthalamic nucleus stimulation. J. Korean Med. Sci. 26, 1344-1355. doi: 10.3346/jkms.2011.26.10.1344

Pinsky, P. F., and Rinzel, J. (1995). Synchrony measures for biological neural networks. Biol. Cybernet. 73, 129-137. doi: 10.1007/BF00204051

Plenz, D., and Kital, S. T. (1999). A basal ganglia pacemaker formed by the subthalamic nucleus and external globus pallidus. Nature 400, 677-682. doi: $10.1038 / 23281$

Plesser, H., Eppler, J., Morrison, A., Diesmann, M., and Gewaltig, M.-O. (2007). "Efficient parallel simulation of large-scale neuronal networks on clusters of multiprocessor computers," in Euro-Par 2007 Parallel Processing, Volume 4641 
of Lecture Notes in Computer Science, eds A.-M. Kermarrec, L. Bougé, and T. Priol (Berlin: Springer), 672-681.

Plesser, H. E., and Austvoll, K. (2009). Specification and generation of structured neuronal network models with the NEST topology module. BMC Neurosci. 10, 1-2. doi: 10.1186/1471-2202-10-S1-P56

Popovych, O. V., Hauptmann, C., and Tass, P. A. (2005). Effective desynchronization by nonlinear delayed feedback. Phys. Rev. Lett. 94:164102. doi: 10.1103/PhysRevLett.94.164102

Popovych, O. V., and Tass, P. A. (2012). Desynchronizing electrical and sensory coordinated reset neuromodulation. Front. Hum. Neurosci. 6:58. doi: 10.3389/fnhum.2012.00058

Popovych, O. V., Yanchuk, S., and Tass, P. A. (2013). Self-organized noise resistance of oscillatory neural networks with spike timing-dependent plasticity. Sci. Rep. 11:2926. doi: 10.1038/srep02926

Pyragas, K., Novicenko, V., and Tass, P. (2013). Mechanism of suppression of sustained neuronal spiking under high-frequency stimulation. Biol. Cybernet. 107, 669-684. doi: 10.1007/s00422-013-0567-1

Ranck, J. B. Jr. (1975). Which elements are excited in electrical stimulation of mammalian central nervous system: a review. Brain Res. 98, 417-440. doi: 10.1016/0006-8993(75)90364-9

Reese, R., Pinsker, M. O., Herzog, J., Wodarg, F., Steigerwald, F., Pötter-Nerger, M., et al. (2012). The atypical subthalamic nucleus-an anatomical variant relevant for stereotactic targeting. Move. Disord. 27, 544-548. doi: 10.1002/mds. 24902

Richardson, K. A., Gluckman, B. J., Weinstein, S. L., Glosch, C. E., Moon, J. B., Gwinn, R. P., et al. (2003). In vivo modulation of hippocampal epileptiform activity with radial electric fields. Epilepsia 44, 768-777. doi: 10.1046/j.15281157.2003.35402.x

Rodriguez-Oroz, M. C., Obeso, J. A., Lang, A. E., Houeto, J.-L., Pollak, P., Rehncrona, S., et al. (2005). Bilateral deep brain stimulation in Parkinson's disease: a multicentre study with 4 years follow-up. Brain 128(Pt 10), 2240-2249. doi: 10.1093/brain/awh571

Rubin, J. E., McIntyre, C. C., Turner, R. S., and Wichmann, T. (2012). Basal ganglia activity patterns in parkinsonism and computational modeling of their downstream effects. Eur. J. Neurosci. 36, 2213-2228. doi: 10.1111/j.14609568.2012.08108.x

Rubin, J. E., and Terman, D. (2004). High frequency stimulation of the subthalamic nucleus eliminates pathological thalamic rhythmicity in a computational model. J. Comput. Neurosci. 16, 211-235. doi: 10.1023/B:JCNS.0000025686.47117.67

Sadek, A. R., Magill, P. J., and Bolam, J. P. (2007). A single-cell analysis of intrinsic connectivity in the rat globus pallidus. J. Neurosci. 27, 6352-6362. doi: 10.1523/JNEUROSCI.0953-07.2007

Schmidt, C., and van Riemen, U. (2012). Modeling the field distribution in deep brain stimulation: the influence of anisotropy of brain tissue. IEEE Trans. Biomed. Eng. 59, 1583-1592. doi: 10.1109/TBME.2012. 2189885

Shen, K.-Z., and Johnson, S. W. (2006). Subthalamic stimulation evokes complex EPSCs in the rat substantia nigra pars reticulata in vitro. J. Physiol. 573, 697-709. doi: 10.1113/jphysiol.2006.110031

Shen, K.-Z., Zhu, Z.-T., Munhall, A., and Johnson, S. W. (2003). Synaptic plasticity in rat subthalamic nucleus induced by high-frequency stimulation. Synapse 50, 314-319. doi: 10.1002/syn.10274

Shink, E., Bevan, M., Bolam, J., and Smith, Y. (1996). The subthalamic nucleus and the external pallidum: two tightly interconnected structures that control the output of the basal ganglia in the monkey. Neuroscience 73, 335-357. doi: 10.1016/0306-4522(96)00022-X

Silchenko, A., Adamchic, I., Hauptmann, C., and Tass, P. A. (2013). Impact of acoustic coordinated reset neuromodulation on effective connectivity in a neural network of phantom sound. Neuroimage 77, 133-147. doi: 10.1016/j.neuroimage.2013.03.013

Smirnov, D. A., Barnikol, U. B., Barnikol, T. T., Bezruchko, B. P., Hauptmann, C., Bührle, C., et al. (2008). The generation of Parkinsonian tremor as revealed by directional coupling analysis. Eur. Lett. 83:20003. doi: 10.1209/02955075/83/20003

Smith, Y., Wichmann, T., Factor, S. A., and DeLong, M. R. (2012). Parkinson's disease therapeutics: new developments and challenges since the introduction of levodopa. Neuropsychopharmacology 37, 213-246. doi: 10.1038/npp. 2011.212
So, R. Q., Kent, A. R., and Grill, W. M. (2012). Relative contributions of local cell and passing fiber activation and silencing to changes in thalamic fidelity during deep brain stimulation and lesioning: a computational modeling study. $J$. Comput. Neurosci. 32, 499-519. doi: 10.1007/s10827-011-0366-4

Steigerwald, F., Pötter, M., Herzog, J., Pinsker, M., Kopper, F., Mehdorn, H., et al. (2008). Neuronal activity of the human subthalamic nucleus in the Parkinsonian and non-Parkinsonian state. J. Neurophysiol. 100, 2515-2524. doi: 10.1152/jn. 90574.2008

Tass, P. A. (1999). Phase Resetting in Medicine and Biology - Stochastic Modelling and Data Analysis. Berlin: Springer. doi: 10.1007/978-3-540-38161-7

Tass, P. A. (2003a). Desynchronization by means of a coordinated reset of neural sub-populations. Prog. Theor. Phys. Suppl. 150, 281-296. doi: 10.1143/PTPS.150.281

Tass, P. A. (2003b). Desynchronization by means of a coordinated reset of neural sub-populations - a novel technique for demand-controlled deep brain stimulation. Prog. Theor. Phys. Suppl. 150, 281-296. doi: 10.1143/PTPS.150.281

Tass, P. A. (2003c). A model of desynchronizing deep brain stimulation with a demand-controlled coordinated reset of neural subpopulations. Biol. Cybernet. 89, 81-88. doi: 10.1007/s00422-003-0425-7

Tass, P. A., Adamchic, I., Freund, H.-J., von Stackelberg, T., and Hauptmann, C. (2012a). Counteracting tinnitus by acoustic coordinated reset neuromodulation. Restor. Neurol. Neurosci. 30, 137-159. doi: 10.3233/RNN-2012-110218

Tass, P. A., Qin, L., Hauptmann, C., Dovero, S., Bezard, E., Boraud, T., et al. (2012b). Coordinated reset has sustained after-effects in Parkinsonian monkeys. Ann. Neurol. 72, 816-820. doi: 10.1002/ana.23663

Tass, P. A., and Hauptmann, C. (2007). Therapeutic modulation of synaptic connectivity with desynchronizing brain stimulation. Int. J. Psychophysiol. 64, 53-61. doi: 10.1016/j.ijpsycho.2006.07.013

Tass, P. A., and Hauptmann, C. (2009). Anti-kindling achieved by stimulation targeting slow synaptic dynamics. Restor. Neurol. Neurosci. 27, 589-609. doi: 10.3233/RNN-2009-0484

Tass, P. A., and Majtanik, M. (2006). Long-term anti-kindling effects of desynchronizing brain stimulation: a theoretical study. Biol. Cybernet. 94, 58-66. doi: 10.1007/s00422-005-0028-6

Tass, P. A., and Popovych, O. (2012). Unlearning tinnitus-related cerebral synchrony with acoustic coordinated reset stimulation: theoretical concept and modelling. Biol. Cybernet. 106, 27-36. doi: 10.1007/s00422-012-0479-5

Tass, P. A., Silchenko, A. N., Hauptmann, C., Barnikol, U. B., and Speckmann, E.-J. (2009). Long-lasting desynchronization in rat hippocampal slice induced by coordinated reset stimulation. Phys. Rev. E 80:011902. doi: 10.1103/PhysRevE.80.011902

Tass, P. A., Smirnov, D. A., Karavaev, A., Barnikol, U. B., Barnikol, T. T., Adamchic, I., et al. (2010). The causal relationship between subcortical local field potential oscillations and Parkinsonian resting tremor. J. Neural Eng. 7:016009. doi: 10.1088/1741-2560/7/1/016009

Terman, D., Rubin, J., Yew, A., and Wilson, C. (2002). Activity patterns in a model for the subthalamopallidal network of the basal ganglia. J. Neurosci. 22, 2963-2976.

van Rossum, M. V. W., Bi, G. Q., and Turrigiano, G. G. (2000). Stable hebbian learning from spike timing-dependent plasticity. J. Neurosci. 20, 8812-8821.

Voges, J., Volkmann, J., Allert, N., Lehrke, R., Koulousakis, A., Freund, H.J., et al. (2002). Bilateral high-frequency stimulation in the subthalamic nucleus for the treatment of Parkinson disease: correlation of therapeutic effect with anatomical electrode position. J. Neurosurg. 96, 269-279. doi: 10.3171/jns.2002.96.2.0269

Volkmann, J. (2004). Deep brain stimulation for the treatment of Parkinson's disease. J. Clin. Neurophysiol. 21, 6-17. doi: 10.1097/00004691-200401000-00003

Volkmann, J., Herzog, J., Kopper, F., and Deuschl, G. (2002). Introduction to the programming of deep brain stimulators. Move. Disord. 17, 181-187. doi: $10.1002 / \mathrm{mds} .10162$

Wichmann, T., DeLong, M. R., Guridi, J., and Obeso, J. A. (2011). Milestones in research on the pathophysiology of Parkinson's disease. Move. Disord. 26, 1032-1041. doi: 10.1002/mds.23695

Wilkinson, B., and Allen, M. (2004). Parallel Programming: Techniques and Applications using Networked Workstations and Parallel Computers. Upper Saddle River, NJ: Prentice Hall.

Wongsarnpigoon, A., and Grill, W. M. (2010). Energy-efficient waveform shapes for neural stimulation revealed with a genetic algorithm. J. Neural Eng. 7:046009. doi: 10.1088/1741-2560/7/4/046009 
Yelnik, J., Percheron, G., and Francois, C. (1984). A golgi analysis of the primate globus pallidus. ii. Quantitative morphology and spatial orientation of dendritic arborizations. J. Comp. Neurol. 227, 200-213. doi: 10.1002/cne. 902270206

Conflict of Interest Statement: Peter Tass is employed by Jülich Research Center and works as Consulting Professor at Stanford University; formerly working with ANM GmbH (Cologne, Germany), shareholder of ANM GmbH. Christian Hauptmann is employed by Jülich Research Center; formerly working with ANM $\mathrm{GmbH}$ (Cologne, Germany). Several patents protect electrical CR neuromodulation. The inventor of this patent is Peter Tass, and the assignee is Jülich Research Center. Martin Ebert reports no potential conflicts of interest.
Received: 06 June 2014; accepted: 05 November 2014; published online: 27 November 2014.

Citation: Ebert M, Hauptmann C and Tass PA (2014) Coordinated reset stimulation in a large-scale model of the STN-GPe circuit. Front. Comput. Neurosci. 8:154. doi: 10.3389/fncom.2014.00154

This article was submitted to the journal Frontiers in Computational Neuroscience. Copyright (C) 2014 Ebert, Hauptmann and Tass. This is an open-access article distributed under the terms of the Creative Commons Attribution License (CC BY). The use, distribution or reproduction in other forums is permitted, provided the original author(s) or licensor are credited and that the original publication in this journal is cited, in accordance with accepted academic practice. No use, distribution or reproduction is permitted which does not comply with these terms. 